McGill-01/26, UW/PT 01-27, hep-th/0201082

\title{
Loop-Generated Bounds on Changes to the Graviton Dispersion Relation
}

\author{
C.P. Burgess ${ }^{a}$ J. Cline, ${ }^{a}$, E. Filotas ${ }^{a}$, J. Matias $^{b}{ }^{b}$ and G.D. Moore ${ }^{c}$ \\ a Physics Department, McGill University, 3600 University Street, Montréal, Québec, Canada H3A 2 T8. \\ ${ }^{b}$ IFAE, Universitat Autònoma de Barcelona, Spain. \\ ${ }^{c}$ Department of Physics, University of Washington, Seattle, WA 98195, USA.
}

\begin{abstract}
We identify the effective theory appropriate to the propagation of massless bulk fields in braneworld scenarios, to show that the dominant low-energy effect of asymmetric warping in the bulk is to modify the dispersion relation of the effective 4-dimensional modes. We show how such changes to the graviton dispersion relation may be bounded through the effects they imply, through loops, for the propagation of standard model particles. We compute these bounds and show that they provide, in some cases, the strongest constraints on nonstandard gravitational dispersions. The bounds obtained in this way are the strongest for the fewest extra dimensions and when the extradimensional Planck mass is the smallest. Although the best bounds come for warped 5-D scenarios, for which $M_{5}$ is $O(\mathrm{TeV})$, even in 4 dimensions the graviton loop can lead to a bound on the graviton speed which is comparable with other constraints.
\end{abstract}

PACS numbers: 11.10.Kk, 04.50.+h

\section{INTRODUCTION}

Although physics in more than the traditional four dimensions has been long speculated to be important for describing phenomena at energies above the electroweak scale, the idea is presently enjoying additional scrutiny because of two more recent developments.

First came the understanding of the strong-coupling limit of string theory in terms of 11-dimensional supergravity interacting on spaces with boundaries [1]. Together with the realization that observable particles can be trapped on these boundaries, or on D-branes, this understanding freed the string scale from the Planck mass, allowing it to be as low as the weak scale [2] or at intermediate or grand-unified scales [3]. Such low string scales allow extra dimensions to be comparatively large, and so (potentially) to have much richer implications at experimentally accessible energies.

Second came the observation that even very small extra dimensions might also have interesting low-energy implications if their geometries are 'warped' [4, 5]. In this framework, the four-dimensional metric has a nontrivial dependence on position in the extra dimensions, allowing four-dimensional properties like masses and couplings to depend in an interesting way on an observer's position within the extra dimensions.

Although not required by either approach, the broader class of geometries which are allowed once observable particles are confined to a brane includes many configurations which violate Lorentz invariance within the observable four dimensions by picking out a preferred frame [6.7], such as by having gravitating objects displaced from our brane in the extra dimensions. For example, within the warped framework one can have a 5D line element of the form

$$
d s^{2}=-(\alpha+\Delta) d t^{2}+\alpha d \vec{x}^{2}+\frac{d r^{2}}{\alpha+\Delta}
$$

where $t, \vec{x}$ are 4 dimensional time and space, $r$ is the extra dimension, $\alpha(r)=r^{2} / l^{2}$ and $\Delta(r)=-\mu / r^{2}+Q^{2} / r^{4}$. If $\mu=Q=0$ this is anti-de Sitter space with curvature radius $l$, and there is no Lorentz violation. But if $\mu \neq 0$, it is the AdS-Reissner-Nördstrom metric with a singularity at $r=0$ and a speed of light which varies with $r$ as $c^{2}(r)=1+\frac{\Delta}{\alpha}$. There is a preferred frame with 4 -velocity $u^{\mu}=(1,0,0,0)$ in these coordinates. In this example, the preferred frame is not due to visible matter, but rather the presence of a "black brane" at $r=0$ which is displaced from our brane in the extra dimension. A priori its effects need not be small, and could cause observable phenomena.

Very strong limits exist on the size of various Lorentzviolating effects involving ordinary particles [8.9], so one expects these to provide the most stringent tests of any Lorentz-violation effects predicted in the brane-world scenario. Although this expectation is borne out, the surprise in these scenarios is always the comparative difficulty of finding good constraints in the event that the only known particle living in the bulk is the graviton, since so little is directly known about the graviton's properties. Some comparatively weak limits do exist, coming from Parameterized Post-Newtonian tests of General Relativity within the solar system [10,11, and from its success in describing the energy loss of the binary pulsar, $1913+16$ [11] 13].

Much stronger limits on changes to the graviton dispersion relation may also be obtained if they permit highenergy particles to Čerenkov radiate gravitons. In this case bounds may be obtained from our observation of very high energy particles in cosmic rays. Assuming these cosmic rays to be protons - as the best evidence indicates [14] - the constraint $\left(c_{p}-c_{g}\right) / c_{p}<10^{-15}$ was obtained 
in this way [15], strongly restricting the case where the graviton velocity, $c_{g}$, is smaller than that of the proton, $c_{p}$.

Our purpose in this paper is to provide a complementary constraint for the case where protons cannot radiate gravitons, and so the above bound does not apply. We obtain our constraint by computing some of the Lorentzviolating effects which are implied for fermions and photons by radiative corrections involving gravitons. Because the bounds on such corrections for electrons and photons are extremely good, we are able to infer reasonably strong bounds on Lorentz violation in the graviton sector.

We find two kinds of effects, each of which depends on physics at different scales. We find the one-loop gravitoninduced contributions to particle phase velocities to be highly suppressed by the mass of the particle involved. This sensitivity is stronger the higher the number of dimensions the graviton sees. The photon and electron are particularly insensitive to this kind of correction. Because they are so mass-dependent, the largest contributions to light particles are often due to higher-loop graphs within which a one-loop graviton-induced modification to the propagation of a heavy particle (like a $W$ boson or top quark) is inserted. This makes predictions sensitive to the ultraviolet structure of the theory.

Graviton loops also change light-particle dispersion relations by introducing contributions which involve higher powers of the particle momentum. We compute these and find that they can be generically less ultraviolet sensitive than are changes to particle phase velocities. Interestingly, the low-energy contributions of this form (i.e. the contributions due to the massless $4 \mathrm{D}$ graviton) are already large enough to provide interesting bounds.

We organize our presentation as follows. In the next section we discuss in general terms how Lorentz-violating effects enter into the low-energy 4D theory at energies well below the compactification scale. This is the regime of interest for experiments, and is not the regime in which one can consider bulk particles to be moving on ballistic trajectories through the extra dimensions. Then in section III we discuss the fermion and photon self energies in general, to identify which features are required in order to make comparison with experiments. Section IV follows with the calculation of the graviton loop, and the derivation of the induced Lorentz violation amongst visible brane-based particles. Given the mass dependence of the results of Section IV, Section V gives conservative estimates as to the size which might be expected for photons, electrons and protons. Section VI describes the bounds on Lorentz violation for these particles, and computes the bound which may be inferred indirectly on the strength of Lorentz violation in the gravity sector. Our conclusions are summarized in section VI, and some of the cumbersome intermediate results are gathered into two appendices.

\section{THE 4D EFFECTIVE PICTURE}

In the brane-world picture photons and electrons usually are constrained to move on our four-dimensional brane, while gravitons are free to explore the higherdimensional bulk surrounding space. It is intuitive in this kind of picture that brane and bulk particles might propagate differently, since bulk particles might be free to take 'shortcuts' through the extra dimensions which are forbidden to brane-bound particles [16]. This possibility has been proposed to be, in some instances, a virtue in that it may provide a novel way to address some cosmological problems 7.17 .

Although this kind of ballistic picture is appealingly intuitive, it is not really appropriate for the low energies at which tests of the dispersion relation actually take place. Experimental tests only involve the one graviton which we see in 4 dimensions, and so only involve the very lowest Kaluza-Klein (KK) state. By contrast, assigning the graviton localized trajectories in the extra dimensions presupposes a localized wave packet in these dimensions, which cannot be constructed purely from the lowest-energy mode.

We briefly detour in this section to describe how extra-dimensional Lorentz-violation appears in the lowenergy effective Lagrangian which describes the lowest KK mode. Although we start by using the simplest example of a scalar field, the conclusions we draw will be shown to be equally valid for higher spin fields.

\section{A. A Scalar Field}

Consider, therefore, a bulk scalar field, $\Phi(x, y)$, where $x^{\mu}$ labels the 4 dimensions parallel to the brane, and $y^{m}$ labels the $n$ various transverse dimensions. The fourdimensional field content is obtained by resolving $\Phi$ into a basis of modes in the extra dimensions:

$$
\Phi(x, y)=\sum_{k} \varphi_{k}(x) u_{k}(y)
$$

where the basis functions, $u_{k}(y)$, are eigenfunctions of the appropriate kinetic/mass operator: $\Delta u_{k}=\omega_{k} u_{k}$.

The kinetic $4 \mathrm{D}$ action for the KK modes, $\varphi_{k}(x)$, is obtained by inserting the mode expansion into the higherdimensional action and integrating the result over the extra dimensions. Using an assumed form for the extradimensional background metric:

$$
\mathcal{G}_{M N}=\left(\begin{array}{cc}
g_{\mu \nu}(x, y) & 0 \\
0 & h_{m n}(y)
\end{array}\right)
$$

one finds in this way:

$$
\begin{aligned}
S & =-\frac{1}{2} \int d^{4} x d^{n} y \sqrt{-\mathcal{G}} \mathcal{G}^{M N} \partial_{M} \Phi \partial_{N} \Phi \\
& =-\frac{1}{2} \int d^{4} x \sqrt{-G} G_{k l}^{\mu \nu} \partial_{\mu} \varphi_{k} \partial_{\nu} \varphi_{l}+\cdots
\end{aligned}
$$


where $\mathcal{G}=\operatorname{det} \mathcal{G}_{M N}$, etc., and the effective four dimensional metric governing the kinetic terms is

$$
\sqrt{-G} G_{k l}^{\mu \nu}(x):=\int d^{n} y \sqrt{-g h} g^{\mu \nu}(x, y) u_{k}^{*}(y) u_{l}(y) .
$$

The main point is that the metrics (plural since there is a different metric for each choice of the indices $k, l$ ) defined by eq. (5) differ, in general, from the induced metric on the brane, $\gamma_{\mu \nu}$, which appears in the kinetic term for fields which are trapped on a brane. For instance, for a brane defined by the surface $y=y_{0}$ the induced metric is simply $\gamma_{\mu \nu}=g_{\mu \nu}\left(x, y_{0}\right)$. This is ultimately the source of Lorentz-violating effects due to the bulk metric.

If we focus purely on the massless four-dimensional mode, which we label by $k=0$, then we must integrate out the other, more massive, KK modes. The kinetic term for the massless field, $\varphi=\varphi_{0}(x)$, contains the metric $G^{\mu \nu}=G_{00}^{\mu \nu}$ which may differ from the induced metric on the brane, $\gamma^{\mu \nu}$. In the rest of this paper we will focus on the implications of this difference.

Two limits of the metric $G^{\mu \nu}$ bear highlighting. First, in the absence of warping of the bulk metric (i.e., if $g^{\mu \nu}(x)$ is independent of $y$ ) eq. (5) becomes:

$$
\begin{aligned}
\sqrt{-G} G_{k l}^{\mu \nu} & =\sqrt{-g} g^{\mu \nu}(x) \int d^{n} y \sqrt{h} u_{k}^{*} u_{l} \\
& =\delta_{k l} \sqrt{-g} g^{\mu \nu}(x)
\end{aligned}
$$

where the second line uses the orthonormality condition of the basis modes, $u_{k}(y)$. In this case bulk and brane modes see the same metric, for branes defined by surfaces $y=y_{0}$.

Second, in the absence of a preferred frame in the bulk metric (such as the AdS metric used by Randall and Sundrum) the metrics $G_{\mu \nu}$ and $\gamma_{\mu \nu}$ must be conformal to one another (i.e., $G_{\mu \nu}(x)=f(x) \gamma_{\mu \nu}(x)$ ), since Lorentz invariance implies they must both locally be proportional to the Minkowski space metric $\eta_{\mu \nu}$.

\section{B. The 4D Graviton}

A similar story gives the influence of Lorentz-violating effects on the propagation of low-energy gravitons within the effective theory below the compactification scale (or the AdS curvature scale, in the case of RS-II-like models without compactification (5]), although the details are a bit more complicated due to gauge invariance. Our purpose here is to identify the leading contributions of Lorentz violations in the higher-energy theory to graviton propagation in the low-energy $4 \mathrm{D}$ theory, and to show that they always may be cast in terms of an appropriate shift of the background metric. .

We assume: $(i)$ our interest is in energies very low compared to the compactification scale, allowing a treatment in terms of the low-energy 4D effective theory; (ii) there is only a single massless spin-2 graviton mode in this effective theory, and (iii) that the dominant effect of the higher-dimensional theory is to break Lorentz invariance but not translation invariance or rotational invariance (in the preferred frame). Under these circumstances the 4D effective theory involves an effective 4D metric field coupled to an order parameter, $u_{\mu}$, which defines the preferred frame. The assumptions of unbroken rotational invariance imply $u_{\mu}$ is timelike, and we rescale it so that it is normalized, satisfying $g^{\mu \nu} u_{\mu} u_{\nu}=-1$.

On grounds of general covariance, the $4 \mathrm{D}$ effective theory with the fewest derivatives has the form: if

$$
\mathcal{L}=-\frac{1}{2 \kappa_{4}^{2}} \sqrt{-g}\left[R+a R^{\mu \nu} u_{\mu} u_{\nu}\right]+\ldots,
$$

for some dimensionless constant $a$. Here the ellipses indicate higher-derivative terms, and we have not written a cosmological constant term, which we assume to be negligible. (We shed no light in this paper on the vexing cosmological constant problem.) Here $\kappa_{4}^{2}=8 \pi G_{4}=1 / M_{4}^{2}$, where $G_{4}$ and $M_{4}$ are the usual $4 \mathrm{D}$ Newton's constant and (rationalized) Planck mass: $M_{4} \sim 2 \times 10^{18} \mathrm{GeV}$. In order of magnitude we expect $a \sim \kappa_{4}^{2} \Lambda^{2}$, where $\Lambda$ is the scale associated with the Lorentz-violating effects at higher energies. Clearly $a$ is naturally very small to the extent that $\Lambda$ is much smaller than the $4 \mathrm{D}$ Planck scale.

The main point now follows. The second term of eq. (7) may be completely absorbed by performing the following field redefinition:

$$
g_{\mu \nu} \rightarrow g_{\mu \nu}-\frac{a}{2}\left[g_{\mu \nu}+2 u_{\mu} u_{\nu}\right]
$$

After having performed this redefinition (and a constant rescaling of the metric), graviton fluctuations about flat space are described performing the expansion $g_{\mu \nu}(x)=$ $G_{\mu \nu}+2 \kappa_{4} h_{\mu \nu}(x)$, in eq. (7), using

$$
G_{\mu \nu}=\eta_{\mu \nu}-\delta c_{g}^{2} u_{\mu} u_{\nu}
$$

where $\eta_{\mu \nu}=\operatorname{diag}(-1,1,1,1)$ is the usual Minkowski metric, $u_{\mu}=\eta_{\mu \nu} u^{\nu}$, and $\delta c_{g}^{2}$ is a small quantity which we shall see has the interpretation as a change in the maximum propagation speed, $c_{g}$, of the graviton. The field

\footnotetext{
${ }^{*}$ We thank M. Pospelov for helpful conversations on this point.

†Our metric is 'mostly plus', and we follow Weinberg's curvature conventions [18]. Momentum 4-vectors are upper case $(P)$, the spatial vector is boldface $(\mathbf{p})$, the magnitude of the spatial piece is lowercase $(p)$.
} 
$h_{\mu \nu}$ here is the canonically-normalized field describing graviton propagation.

Just as was the case for the scalar field, the leading effect of higher-energy Lorentz violation is in this way seen to be a modification of the background metric through which the graviton propagates.

As a concrete illustration of this general argument, we can compute the effective 4-D gravitational metric corresponding to eq. (1), treating the Lorentz-violating term as a perturbation. The $4 \mathrm{D}$ effective gravitational action can be obtained by expanding the $5 \mathrm{D}$ action $S$ to linear order in $\delta g_{\mu \nu}=\left(-\mu / r^{2}+Q^{2} / r^{4}\right) u_{\mu} u_{\nu}$, and integrating over the extra dimension. If we write

$$
d s^{2} \cong \frac{r^{2}}{l^{2}}\left(g_{\mu \nu}(x)-\frac{l^{2}}{r^{2}} \delta g_{\mu \nu}\right) d x^{\mu} d x^{\nu}+\frac{l^{2}}{r^{2}} d r^{2}
$$

(the correction $\Delta(r)$ in $g_{r r}$ can be neglected to leading order in $\Delta$ ) then

$$
\begin{aligned}
\delta S= & \frac{1}{2} M_{5}^{3}\left(\int_{r_{1}}^{r_{2}} d r \frac{r}{l} \cdot \frac{l^{2}}{r^{2}}\left[-\frac{\mu}{r^{2}}+\frac{Q^{2}}{r^{4}}\right]\right) \\
& \times \sqrt{-g}\left(R^{\mu \nu}-\frac{1}{2} g^{\mu \nu} R\right) u_{\mu} u_{\nu}
\end{aligned}
$$

The upper limit of integration corresponds to the position of one brane, and the lower limit might be that of another brane, or else the position of an event horizon where $\alpha\left(r_{1}\right)+\Delta\left(r_{1}\right)=0$, if there is only a single brane. Comparing to the preceding discussion, we see that the graviton sees a metric of the form (9), with

$$
\delta c_{g}^{2} \sim \frac{\int_{r_{1}}^{r_{2}} d r \frac{l}{r}\left[-\frac{\mu}{r^{2}}+\frac{Q^{2}}{r^{4}}\right]}{\int_{r_{1}}^{r_{2}} d r \frac{r}{l}}
$$

Interestingly, the sign of $\delta c_{g}^{2}$ can be positive or negative, depending on the relative sizes of black brane mass and charge.

\section{Physical Implications}

The physical significance of the metric, $G_{\mu \nu}$, appearing in the kinetic term of a field within the effective theory, is most easily seen by working within the geometrical-optics approximation. Within this approximation, the propagating field is written in the form $\varphi(x)=A(x) \exp \left[i P_{\mu} x^{\mu}\right]$, with $A(x)$ assumed to be much more slowly-varying than is the phase, $P_{\mu} x^{\mu}$. With this

\footnotetext{
${ }^{\ddagger}$ For a warped metric of the form $d s^{2}=a(r) g_{\mu \nu} d x^{\mu} d x^{\nu}+$ $b(r) d r^{2}$, the reduction of the gravitational action from $5 \mathrm{D}$ to $4 \mathrm{D}$ is $S=-\frac{1}{2} M_{5}^{3}\left(\int a \sqrt{b} d r\right) \sqrt{-g} R$, where $R$ is the Ricci scalar constructed from the $4 \mathrm{D}$ part of the metric, $g_{\mu \nu}$.
}

choice the field equation, $\left(G^{\mu \nu} \nabla_{\mu} \nabla_{\nu}-m^{2}\right) \varphi=0$ is equivalent to the dispersion relation: $G^{\mu \nu} P_{\mu} P_{\nu}+m^{2} \approx 0$, or equivalently the normal vectors, $P_{\mu}$, of the surfaces of constant phase are timelike (or null, if $m=0$ ) vectors of the metric $G^{\mu \nu}$.

If the (four-dimensional) wavelength of the mode is much smaller than the (four-dimensional) radius of curvature of the background fields, then the motion of these wave packets is along the geodesics of the $4 \mathrm{D}$-metric $G_{\mu \nu}$. Clearly these trajectories and dispersion relations generically differ for fields which have different metrics in their kinetic terms.

The statement is slightly weaker for massless particles, since the latter move along null geodesics of their respective metrics. Consequently, their trajectories only differ if the two metrics are not conformal to one another. In particular, differences in the trajectories of massless particles are not observable (in the geometric-optics limit) in the absence of a preferred frame in the bulk or on the brane.

\section{LOOPS: GENERAL CONSIDERATIONS}

We now turn to the general implications for fermions and photons of loop-generated Lorentz-violating effects. Motivated by the considerations of the previous section, we imagine from here on that all brane fields - i.e., all experimentally observed elementary particles except for the graviton - see only the induced metric on the brane, which we take to be flat and Lorentz invariant: $\gamma_{\mu \nu}=\eta_{\mu \nu}=\operatorname{diag}(-1,1,1,1)$. By contrast, the metric appearing within the kinetic terms of any low-energy bulk fields - which we take to be just the graviton - involves the Lorentz-violating metric of eq. (9). This is the dominant low-energy source of Lorentz violation in the effective theory, and it is the only type of Lorentz violation whose implications we shall follow.

In general, loops involving virtual bulk states communicate the news of Lorentz violation to the brane fields, and our task is to compute the size of this effect. In this section we address general issues which follow purely from the assumption that $G^{\mu \nu}=\operatorname{diag}\left(-1 / c_{g}^{2}, 1,1,1\right)$ encodes all Lorentz-violating effects, and return in later sections to the explicit calculation of these effects from graviton loops.

Since we know from direct bounds that Lorentzviolating bulk effects are small (more about this later), we take $c_{g}=1+\epsilon$ with $\epsilon \ll 1$. Because of the very strong constraints already known for $c_{g}<1$ [15] our primary interest in what follows is in positive $\epsilon$. In view of the direct bounds arising from solar-system and binary-pulsar tests 
of general relativity we imagine $\epsilon \lesssim 10^{-6}$.

\section{A. Photon Propagation}

We identify in this section those parts of the gravitoninduced vacuum polarization which have implications for the dispersion relation of transversely-polarized photons.

We first write the most general form for the vacuum polarization which can be built from the tensors $\eta_{\mu \nu}$, $G_{\mu \nu}=\eta_{\mu \nu}+\left(1-c_{g}^{2}\right) u_{\mu} u_{\nu}$ and the momentum 4-vector, $P_{\mu}$, which is consistent with symmetry $\left(\Pi^{\mu \nu}=\Pi^{\nu \mu}\right)$ and transversality $\left(P_{\mu} \Pi^{\mu \nu}=0\right)$. The most general form is:

$$
\begin{aligned}
\Pi^{\mu \nu}=A & \left(\eta^{\mu \nu}-\frac{P^{\mu} P^{\nu}}{P^{2}}\right)+B\left[u^{\mu} u^{\nu}\right. \\
& \left.+\frac{(P \cdot u)^{2}}{P^{4}} P^{\mu} P^{\nu}-\frac{(P \cdot u)}{P^{2}}\left(P^{\mu} u^{\nu}+P^{\nu} u^{\mu}\right)\right],
\end{aligned}
$$

where at this point $A$ and $B$ are arbitrary functions of the two independent variables, $P^{2}$ and $P \cdot u$.

The dispersion relation is found by searching for the zero eigenvalues of the inverse propagator, $\Delta^{\mu \nu}=\Delta_{0}^{\mu \nu}+$ $\Pi^{\mu \nu}$ where $\Delta_{0}^{\mu \nu}=-\left(P^{2} \eta^{\mu \nu}-P^{\mu} P^{\nu}\right)$. In particular, our interest is only in those which are transverse (orthogonal to the pure gauge directions). Working in the rest frame, $u^{\mu}=(1,0,0,0)$, and taking the photon momentum to point in the $z$-direction (i.e., $P^{\mu}=(\omega, 0,0, p)$ ), we therefore require that $\Delta^{\mu \nu}=0$ in the directions $\mu, \nu=x, y$.

A simple calculation using eq. (13) shows that this implies:

$$
\omega^{2}-p^{2}+A(\omega, p)=0 .
$$

Since $A$ is perturbatively small, it suffices to write the dispersion relation as $\omega=\omega_{0}+\omega_{1}$, where $\omega_{0}=p$, and to evaluate $A$ with $\omega=\omega_{0}$. This leads to the present section's main result:

$$
\omega_{1}=-\frac{1}{2 \omega_{0}} A\left(\omega_{0}, p\right) .
$$

As we shall find, $A$ admits an expansion at low energies in powers of $P^{2}$ and $u \cdot P$, leading to the form

$$
A\left(\omega_{0}, p\right)=\alpha p^{2}+\beta p^{4}+\ldots,
$$

\footnotetext{
${ }^{\S} \mathrm{A}$ weaker bound, $\epsilon \lesssim 10^{-3}$, is required if only terrestrial bounds, or the gravitational radiation rate of the binary pulsar are used. The stronger limit follows from angular momentum conservation for the Sun, as inferred by requiring the ecliptic and solar equatorial planes not to precess relative to one another throughout the history of the solar system [19, 10,11.
}

where rotational invariance precludes the appearance of odd powers of $p$. Using this in eq. (15) and comparing the result with the general $4 \mathrm{D}$ photon dispersion relation

$$
\omega^{2}(p)=p^{2} c_{\gamma}^{2}+b_{\gamma} p^{4}+O\left(p^{6}\right) .
$$

we readily identify

$$
c_{\gamma}^{2}=1-\alpha, \quad b_{\gamma}=-\beta .
$$

\section{B. Fermion Propagation}

We next ask how loop contributions to fermion selfenergies can modify fermion dispersion relations. We work within the rest frame defined by $u^{\mu}$. Writing the inverse electron propagator as $S=S_{0}+\Sigma$, with $S_{0}=-(i \not P+m)$, we see that to leading order in perturbation theory the zeroes of $S$ satisfy $P^{\mu}=(E, \mathbf{p})$, where $E=E_{0}+E_{1}$ with $E_{0}=\sqrt{p^{2}+m_{0}^{2}}$ and:

$$
i \gamma^{0} E_{1}=-\Sigma\left(E_{0}, \mathbf{p}\right)
$$

For small $\mathbf{p}, \Sigma$ has the expansion

$$
\begin{aligned}
\Sigma\left(E_{0}, \mathbf{p}\right)=\mathcal{A}+ & \mathcal{B}(i \vec{\gamma} \cdot \mathbf{p})+\mathcal{C} p^{2} \\
& +\mathcal{D} p^{2}(i \vec{\gamma} \cdot \mathbf{p})+\mathcal{E} p^{4}+\cdots
\end{aligned}
$$

and so eq. (19) implies a dispersion relation of the form

$$
E_{f}^{2}=m_{f}^{2}+p^{2} c_{f}^{2}+b_{f} p^{4}+\cdots
$$

with

$$
\begin{aligned}
m_{f} & =m_{0}-\mathcal{A}+\cdots \\
c_{f}^{2} & =1-2\left(\mathcal{B}+m_{0} \mathcal{C}\right)+\cdots \\
b_{f} & =-2\left(\mathcal{D}+m_{0} \mathcal{E}\right)+\cdots
\end{aligned}
$$

In these last three equations the subscript $f$ denotes the fermion species, and the ellipses indicate higher-order contributions.

Since it is the quantities $c_{f}^{2}$ and $b_{f}$ which we wish to compare with experiments, the implications of graviton loops may be obtained by computing the coefficients $\mathcal{B}$ through $\mathcal{E}$.

\section{LOOPS: GRAVITON CALCULATIONS}

In this section we compute the one-loop self energy which is obtained when a fermion or photon emits and reabsorbs a virtual graviton, as in figs. 1 and 2. We present our results in three steps. First, since the integrals involved strongly diverge in the ultraviolet, we make some general remarks about the correct way to treat these divergences before describing the calculations themselves. 
We then evaluate the graviton loop in two steps, motivated by the picture that Lorentz violation is arising from field configurations within the extra-dimensional bulk. First we consider loops involving only the lowest KK mode: the massless $4 \mathrm{D}$ graviton. These loops have the virtue of only involving known particles and couplings, and so the results we obtain are comparatively robust. They describe the graviton contributions within the effective $4 \mathrm{D}$ theory, well below the compactification scale, $M_{c} \sim 1 / r$, where $r$ is a measure of the linear size of the extra dimensions. In the case of a single noncompact extra dimension, the effective theory is good below the bulk curvature scale $\sqrt{-\Lambda_{5} / M_{5}^{3}}$, where $\Lambda_{5}$ is the bulk cosmological constant and $M_{5}$ is the 5 -D gravity scale.

Next, we compute the contributions of gravitons in the effective theory between $M_{c}$ and the scale $M_{l}>M_{c}$ associated with the extra-dimensional Lorentz violating physics. Since the theory is extra-dimensional in this energy range, this involves calculating the loop contributions of higher KK graviton modes. In order to do this we make several simplifying assumptions about the nature of the extra-dimensional Lorentz violation, which we believe suffices for the purposes of establishing the order of magnitude of the extra-dimensional result.

\section{A. Ultraviolet Divergences}

In $d$ spacetime dimensions the gravitational coupling has dimension $\kappa_{d} \sim M_{d}^{1-d / 2}$, where $M_{d}$ is the $d$ dimensional Planck mass. On dimensional grounds we therefore expect the most divergent contribution to oneloop brane-particle dispersion relations to be

$$
c^{2}-1 \sim \kappa_{d}^{2} \Lambda^{d-2}, \quad b \sim \kappa_{d}^{2} \Lambda^{d-4},
$$

where $\Lambda$ is the ultraviolet cutoff scale.

As is usual within an effective theory, this indicates that the result is most sensitive to the most energetic degrees of freedom in the problem, suggesting that calculations within the full theory would produce results that are of order $c^{2}-1 \sim \kappa_{d}^{2} M^{d-2}$ and $b \sim \kappa_{d}^{2} M^{d-4}$, where $M$ might be the mass of a heavy particle which was integrated out to produce the low-energy effective theory.

As we shall see, the above mass-dependence is roughly right, although some care is required due to the appearance of power-law divergences [20]. Care is required because, although the renormalization group ensures that the coefficient of large logarithms like $\log (M)$ in observable quantities like $c^{2}-1$ or $b$ may be read off from the coefficients of $\log$ divergences (like $\log (\Lambda)$ ) within the effective theory, the same is not true for higher (powerlaw) divergences. As a result in this section we ignore all power divergences, and compute only the log-divergent parts of the results. If the results do not have log divergences (as will be the case with an odd number of extra dimensions), then we compute only the finite parts of the loops.

Neglecting the power divergences minimizes the Lorentz symmetry violation which is seen by particles on the brane, and so leads to conservative conclusions. It corresponds to considering the theory in which branebound particles respect Lorentz invariance at the energy scale where the theory becomes 4 dimensional. We return in Section V to the issue of contributions which are proportional to positive powers of large mass scales, $M$, by considering higher loops which explicitly involve more massive virtual particles. We shall there see that naive power-counting estimates do correctly reproduce the $M$ dependence of the results, but miss important dimensionless loop factors.

In practice the finite and log-divergent terms are most easily obtained within dimensional regularization, within which power-law divergences do not arise. We have computed our results both using dimensional regularization and using an explicit ultraviolet cutoff, however, and have verified that the answers obtained are the same in both cases.

\section{B. Four-dimensional Graviton}

The Feynman rules for fermions and gravitons in the absence of Lorentz-violating effects are standard, and are obtained by linearizing the Dirac-Einstein-Hilbert action in curved space,

$$
\mathcal{L}=-\sqrt{-g}\left[\frac{1}{2 \kappa_{4}^{2}} R+\bar{\psi}(\not D+m) \psi+\frac{1}{4} F_{\mu \nu} F^{\mu \nu}\right],
$$

about flat space: $g_{\mu \nu}=\eta_{\mu \nu}+2 \kappa_{4} h_{\mu \nu}$. As before $\kappa_{4}$ denotes the rationalized Planck mass in 4D: $M_{4} \sim 10^{18}$ $\mathrm{GeV} . h_{\mu \nu}$ represents the canonically-normalized graviton field. A recent statement of the resulting Feynman rules can be found in references [21,22].

As we have argued in previous sections, we know that the only Lorentz-violating modification to these rules consists in replacing the Minkowski metric, $\eta_{\mu \nu}$, with the nonstandard metric, $G_{\mu \nu}$, when linearizing the first term in eq. (26) to obtain the graviton propagator. In de Donder gauge this gives:

$$
G_{\mu \nu: \alpha \beta}(Q)=\frac{P_{\mu \nu: \alpha \beta}}{G^{\lambda \rho} Q_{\lambda} Q_{\rho}-i \varepsilon},
$$

where

$$
P_{\mu \nu: \alpha \beta}=\frac{1}{2}\left(G_{\mu \alpha} G_{\nu \beta}+G_{\mu \beta} G_{\nu \alpha}-G_{\mu \nu} G_{\alpha \beta}\right),
$$

and $\varepsilon$ - not to be confused with $\epsilon=c_{g}-1$ - is the infinitesimal which ensures the propagator satisfies Feynman boundary conditions. The fermion propagators and 
vertices arise on the brane, and so use only the usual Minkowski metric.

\section{The Photon Vacuum Polarization}

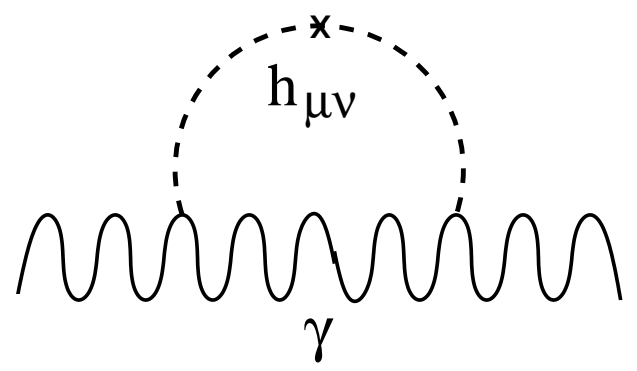

Figure 1. Graviton contribution to photon vacuum polarization.

After Wick rotation, the one-loop Feynman graph in which a virtual graviton is emitted and reabsorbed by the photon (fig. 1) leads to the following expression for the photon vacuum polarization:

$$
\Pi^{\mu \nu}=\left(\frac{\kappa_{4}}{2}\right)^{2} \int \frac{d^{4} Q}{(2 \pi)^{4}} \frac{N^{\mu \nu}}{D},
$$

where $D=(P-Q)^{2} G^{\alpha \beta} Q_{\alpha} Q_{\beta}$ and

$$
N^{\mu \nu}=V^{\mu \lambda: \alpha \beta}(P, P-Q) V_{\lambda}^{\nu: \sigma \rho}(P-Q, P) P_{\alpha \beta: \sigma \rho} .
$$

Appendix A gives explicit expressions for the vertex functions, $V^{\alpha \beta: \mu \nu}(P, Q)$. In these expressions all dependence on the metric $G^{\mu \nu}$ is explicit, and the brane metric, $\eta_{\mu \nu}$, is to be used to perform any implicit index contractions, such as in $(P-Q)^{2}$.

Evaluating this expression (we used the programs FORM and MATHEMATICA to perform the tensor contractions), Taylor expanding in powers of $\epsilon=c_{g}-1$ and performing the momentum integral gives the following expression for the coefficient function, $A$, of eq. (13):

$$
A\left(\omega_{0}, p\right)=\frac{304}{15} \lambda_{4} \epsilon^{2} p^{4}+O\left(\epsilon^{3}\right)
$$

where $\lambda_{4}=\left(\kappa_{4} / 8 \pi\right)^{2} \quad \mathcal{L}$. Here $\mathcal{L}=\log \left(\Lambda^{2} / \mu^{2}\right)=$ $2 /(4-n)$, where the first equality is evaluated using an ultraviolet cutoff, $\Lambda$, and the second regularizes by continuing the spacetime dimension, $n$, away from $4 . \mu$ is an arbitrary scale. We ignore the finite part of the integral relative to its log-divergent part.

Comparison with the general expressions provided earlier gives the following dispersion coefficients:

$$
c_{\gamma}^{2}-1=0, \quad b_{\gamma}=-\frac{304}{15} \lambda_{4} \epsilon^{2} .
$$

\section{The Fermion Self-Energy}

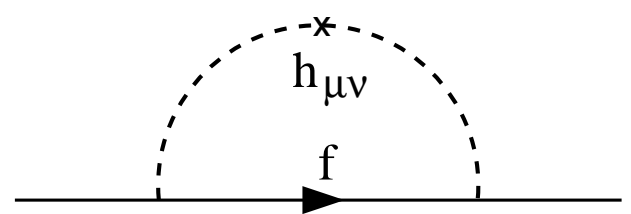

Figure 2. Graviton contribution to fermion self-energy.

We proceed in a similar way for the changes to the fermion self energy. Evaluating the one-loop Feynman graph using the graviton propagator of eq. (27), and Wick-rotating to Euclidean momenta, leads to the following expression for the fermion self energy:

$$
\Sigma_{4}=-\left(\frac{\kappa_{4}}{2}\right)^{2} \int \frac{d^{4} Q}{(2 \pi)^{4}} \frac{N_{1}}{D},
$$

where

$$
\begin{aligned}
& N_{1}=\frac{1}{2} G_{\mu \nu} \gamma^{\mu} {[-i(\not P-\not)+m] \gamma^{\nu} } \\
& \times G_{\alpha \beta}(2 P-Q)^{\alpha}(2 P-Q)^{\beta}, \\
& D=G^{\lambda \rho} P_{\lambda} P_{\rho}\left[(P-Q)^{2}+m^{2}\right] .
\end{aligned}
$$

Again all dependence on the metric $G^{\mu \nu}$ is explicit, and the brane metric, $\eta_{\mu \nu}$, is used to perform the implicit index contractions in $(P-Q)^{2}$ and $\not P$. To derive this we used the simple vertex function $\left(k_{1}+k_{2}\right)_{\mu} \gamma_{\nu}+\left(k_{1}+k_{2}\right)_{\nu} \gamma_{\mu}$ of ref. 21 for the fermion-fermion-graviton coupling rather than the more complicated one of 22, which includes an extra term $\left(i k_{1}+i \not k_{2}+2 m\right) \eta_{\mu \nu}$. The neglect of this extra term is justified - even within loop graphs - because it vanishes if the fermion field equations are used. This ensures that it is an irrelevant operator, in the sense that it can be removed by performing a field redefinition of the fermion of the form $\delta \psi \propto h^{\mu}{ }_{\mu} \psi$.

We have evaluated this integral to the lowest two orders in the small parameter $\epsilon=c_{g}-1$, using the programs FORM/MATHEMATICA to keep track of the various 4vectors in the problem. We find the following results for the logarithmically-divergent part of the coefficients $\mathcal{A}$ through $\mathcal{E}$ of eq. 20 ):

$$
\begin{aligned}
\mathcal{A}_{4} & =m^{3} \lambda_{4}\left(4+13 \epsilon+\frac{33}{2} \epsilon^{2}+\ldots\right), \\
\mathcal{B}_{4} & =m^{2} \lambda_{4}\left(4 \epsilon+10 \epsilon^{2}+\ldots\right), \\
\mathcal{C}_{4} & =\frac{m \lambda_{4}}{3}\left(16 \epsilon+35 \epsilon^{2}+\ldots\right), \\
\mathcal{D}_{4} & =\lambda_{4}\left(6 \epsilon^{2}+\ldots\right), \\
\mathcal{E}_{4} & =0+\ldots
\end{aligned}
$$

where as before $\lambda_{4}=\left(\kappa_{4} / 8 \pi\right)^{2} \mathcal{L}$, with $\mathcal{L}=\log \left(\Lambda^{2} / \mu^{2}\right)=$ $2 /(4-n)$.

These imply the following contributions to the dispersion relation of eq. (21): 


$$
\begin{aligned}
c_{f}^{2}-1 & =-\frac{2 m_{f}^{2} \lambda_{4}}{3}\left[28 \epsilon+65 \epsilon^{2}+\ldots\right], \\
b_{f} & =-\lambda_{4}\left[12 \epsilon^{2}+\ldots\right]
\end{aligned}
$$

For the case of interest, $\epsilon>0$, we see that $c_{f}^{2}<1$ corresponding to fermions propagating slower than light. It follows that (for a given momentum, $p$ ) the fermion energy is depressed compared to the photon energy for small $p$.

Notice that the coefficients $b_{f}$ and $b_{\gamma}$ both first arise at $O\left(\epsilon^{2}\right)$, and so their sign (which is negative for both) does not depend on the sign of $\epsilon$. Furthermore, these terms satisfy $b_{f}>b_{\gamma}$. These terms therefore act to raise the fermion energy relative to the photon, and so act in the opposite direction of the effect of $c_{f}<c_{\gamma}$ (when $\epsilon>0$ ).

\section{Higher-dimensional Gravitons}

We may now estimate the contributions of the higher KK graviton modes to the propagation of brane-based fermions. Rather than doing so by performing a sum over a tower of 4-D KK states, we proceed by directly performing the loop graph using higher-dimensional gravitons.

The first step is to specify what the higher-dimensional metric is about which the graviton fluctuation is to be considered. In principle this should be the metric which describes the gravitational field of the object or objects in the bulk, whose presence gives rise to the preferred frame which violates Lorentz invariance. Since the explicit form for such metrics is rarely known, we proceed by a more approximate route.

Our approximation is based on the observation that higher-dimensional graviton loops are ultraviolet sensitive, with their dominant contributions arising from the circulation of very short-wavelength modes. So long as the wavelength of these modes is much shorter than the radii of curvature of the background Lorentzviolating metric, it should be sufficient to replace the background metric by one which is approximately flat, but Lorentz-violating. In particular, this should be sufficiently accurate for our purposes of estimating the orderof-magnitude of the resulting loop-generated contributions to fermion propagation on the brane.

Accordingly we imagine the higher-dimensional graviton to propagate about a flat metric in which there is a preferred frame defined by an approximately constant $d$-vector, $u^{\mu}$. Such a metric is again described by eq. (9), although with $G_{\mu \nu}$ now being $d$-dimensional.

Linearizing the extra-dimensional Einstein-Hilbert action about this metric $-g_{a b}=G_{a b}+2 \kappa_{d} h_{a b}$ - we find the following $d$-dimensional graviton propagator in de Donder gauge:

$$
G_{\mu \nu: \alpha \beta}=\frac{\frac{1}{2}\left(G_{\mu \alpha} G_{\nu \beta}+G_{\mu \beta} G_{\nu \alpha}\right)-\frac{1}{d-2} G_{\mu \nu} G_{\alpha \beta}}{G^{\lambda \rho} P_{\lambda} P_{\rho}-i \varepsilon} .
$$

Again $\varepsilon$ in this expression is the infinitesimal which enforces Feynman boundary conditions. The quantity $\kappa_{d}$ is related to the $d$-dimensional Newton's constant and Planck mass by $\kappa_{d}^{2}=8 \pi G_{d}=\left(1 / M_{d}\right)^{d-2}$. We take the fermions and photons to move on a 3-brane, for which the induced metric is the usual Minkowski metric.

\section{The Photon Vacuum Polarization}

Computing the photon vacuum polarization with this propagator gives no correction to the photon dispersion relation at low energies, if the number of dimensions exceeds the usual four. This can be understood purely in terms of dimensional analysis, and the fact that we are computing only the finite or log-divergent contributions. The one-loop contributions to the photon selfenergy have two graviton vertices, so they are proportional to $\kappa_{d}^{2}=M_{d}^{-(d-2)}$. Since the log-divergent and finite parts do not involve powers of the cutoff $\Lambda$, the only quantity which can be used to make a dimensionally correct answer is the photon momentum, $p$. Thus the result is proportional to $\kappa_{d}^{2} p^{d}$. This gives a $p^{4}$ correction to the dispersion relation, but only if $d=4$. For $d>4$ the correction always gives only a higher than quartic power of $p$. (Section $\mathrm{V}$ discusses the physics of the power-divergent contributions to $c_{\gamma}^{2}-1$ and $b_{\gamma}$.)

\section{The Fermion Self-Energy}

Evaluating the one-loop fermion self-energy graph using the $d$-dimensional graviton propagator of eq. (37), and Wick-rotating to Euclidean momenta, leads to the following expression for the fermion self energy:

$$
\Sigma_{d}=-\left(\frac{\kappa_{d}}{2}\right)^{2} \int \frac{d^{d} Q}{(2 \pi)^{d}}\left(\frac{N_{1}+N_{2}}{D}\right),
$$

where $N_{1}$ is as given by eq. (34) and

$$
\begin{aligned}
N_{2}= & C_{d}\left\{[i(\not P-\not)+m] G_{\mu \nu}(2 P-Q)^{\mu}(2 P-Q)^{\nu}\right. \\
& \left.-2 i G_{\alpha \beta} \gamma^{\alpha}(2 P-Q)^{\beta} G_{\lambda \rho}(P-Q)^{\lambda}(2 P-Q)^{\rho}\right\},
\end{aligned}
$$

with $C_{d}=(d-4) /[2(d-2)]$.

Evaluating the integral in powers of $\epsilon=c_{g}-1$ gives an ultraviolet divergent result. We have evaluated the result using both dimensional regularization and an explicit cutoff, and have verified that the coefficients of the logarithmically-divergent and pole terms are the same in both cases. For odd dimensions the integrals are finite in 
dimensional regularization, and we have verified that the result agrees with the finite part when evaluated with an ultraviolet cutoff.

We are led in this way to the following expressions for the quantity $c_{f}^{2}-1$ for dimensions $d=5$ through 10 . (We give the quantities $\mathcal{A}$ through $\mathcal{E}$ in Appendix B.):

$$
\begin{aligned}
c_{f}^{2}-1 & =m_{f}^{3} \lambda_{5}\left[\frac{110}{9} \epsilon+\frac{239}{9} \epsilon^{2}+\ldots\right], & & (d=5) \\
& =m_{f}^{4} \lambda_{6}\left[\frac{48}{5} \epsilon+\frac{99}{5} \epsilon^{2}+\ldots\right], & & (d=6) \\
& =-m_{f}^{5} \lambda_{7}\left[\frac{616}{75} \epsilon+\frac{244}{15} \epsilon^{2}+\ldots\right], & & (d=7) \\
& =-m_{f}^{6} \lambda_{8}\left[\frac{464}{63} \epsilon+\frac{890}{63} \epsilon^{2}+\ldots\right], & & (d=8) \\
& =m_{f}^{7} \lambda_{9}\left[\frac{333}{49} \epsilon+\frac{1245}{98} \epsilon^{2}+\ldots\right], & & (d=9) \\
& =m_{f}^{8} \lambda_{10}\left[\frac{115}{18} \epsilon+\frac{421}{36} \epsilon^{2}+\ldots\right], & & (d=10)
\end{aligned}
$$

Here the quantities $\lambda_{d}$ are defined in terms of the couplings $\kappa_{d}$ by:

$$
\begin{array}{lll}
\lambda_{5}=\frac{\kappa_{5}^{2}}{(8 \pi)^{2}}, & \lambda_{6}=\frac{2 \kappa_{6}^{2}}{(8 \pi)^{3}} \mathcal{L}, & \lambda_{7}=\frac{\kappa_{7}^{2}}{6(4 \pi)^{3}}, \\
\lambda_{8}=\frac{2 \kappa_{8}^{2}}{(8 \pi)^{4}} \mathcal{L}, & \lambda_{9}=\frac{\kappa_{9}^{2}}{15(4 \pi)^{4}}, & \lambda_{10}=\frac{4 \kappa_{10}^{2}}{3(8 \pi)^{5}} \mathcal{L} .
\end{array}
$$

As before $\mathcal{L}=\log \left(\Lambda^{2} / \mu^{2}\right)$ when a cutoff is used, or $\mathcal{L}=$ $2 /(d-n)$ in dimensional continuation of $n$ away from $n=d$.

Notice that the corrections to $c_{f}^{2}$ which are implied in this way are not universal in size for all fermions, being suppressed by powers of $m_{f}$ for lighter fermions.

The corresponding higher-order dispersion coefficient, $b_{f}$, is similarly:

$$
\begin{aligned}
b_{f} & =m_{f} \lambda_{5}\left[\frac{26}{3} \epsilon^{2}+\ldots\right], & & (d=5) \\
& =m_{f}^{2} \lambda_{6}\left[\frac{36}{5} \epsilon^{2}+\ldots\right], & & (d=6) \\
& =-m_{f}^{3} \lambda_{7}\left[\frac{32}{5} \epsilon^{2}+\ldots\right], & & (d=7) \\
& =-m_{f}^{4} \lambda_{8}\left[\frac{124}{21} \epsilon^{2}+\ldots\right], & & (d=8) \\
& =m_{f}^{5} \lambda_{9}\left[\frac{39}{7} \epsilon^{2}+\ldots\right], & & (d=9) \\
& =m_{f}^{6} \lambda_{10}\left[\frac{16}{3} \epsilon^{2}+\ldots\right], & & (d=10) .
\end{aligned}
$$

Just as we saw for $d=4, b_{f}$ always arises at second order in $\epsilon$, and so its sign is completely determined in our calculation. As we shall see, the best bound on this coefficient arises when $b_{f}>b_{\gamma} \approx 0$.
The above expressions were derived specifically with the scenario of large, flat extra dimensions in mind. However we can also interpret at least the $5 \mathrm{D}$ result in terms of a warped extra dimension. This has more interesting consequences than the flat case, where the quantum gravity scale would have to exceed $M_{5} \gtrsim 10^{8} \mathrm{GeV}$ in order to comply with sub-millimeter tests of gravity [23]. In the warped case, even if $M_{5} \sim M_{p}$, the KK gravitons couple to the $\mathrm{TeV}$ brane with $\mathrm{TeV}$ strength, and they have a mass gap of order $\mathrm{TeV}$. In this model, we live on a "TeV brane" at $r=r_{1}$, displaced from the "Planck brane" at $r=r_{2}$ such that $\frac{r_{1}}{r_{2}} \sim 10^{-16}$ in accordance with solving the hierarchy problem. As far as the contributions from the ultraviolet graviton loops are concerned, this looks like quantum gravity with a scale of $M_{5} \sim \mathrm{TeV}$. The TeV mass gap protects low energy gravity from any observable distortion, but not so the loop effects from momenta $p \gg \mathrm{TeV}$.

\section{REAL-WORLD COMPLICATIONS}

A further step is required before these results can be compared with the experimental limits on the dispersion relations of real particles. This step involves identifying which low-energy particles produce the largest contributions to any given dispersion relation, **

There are two reasons why this additional step is required. First, we would like to apply the above calculations to protons, for which the experimental limits are the strongest. Unfortunately, our calculations treat all fermions as elementary, and so can only apply directly to the proton for scales below roughly $m_{p} \sim 1 \mathrm{GeV}$. (Notice these low energies may nonetheless be described by an extra-dimensional effective theory within ADD-type scenarios.) For higher energies, we must apply our calculations to the constituent quarks and gluons, and infer from these how the proton dispersion is affected. Unfortunately, the resulting strong-interaction uncertainties prohibit us from following in more detail the $O(1)$ factors and signs of the results, limiting us to an order-ofmagnitude analysis for the proton.

The second reason for being careful in applying our results is the strong dependence which they have on the relevant fermion masses. In particular, it may be that larger contributions are obtained for light particles (like electrons and photons) by embedding the graviton-induced Lorentz-violating contributions of heavier particles (like top quarks) within additional loops. This is how we will recover the larger contributions that might have naively

\footnotetext{
${ }^{* *}$ We thank M. Pospelov for making many very useful suggestions for this and the next sections.
} 
been obtained by keeping the power divergences of the graviton loop graphs.

\section{A. Photons}

Our result for the photon dispersion relation is particularly simple, with $c_{\gamma}=1$ for all $d$, and $b_{\gamma} \neq 0$ only for $d=4$. This simplicity is largely due to our concentrating on the finite and log-divergent contributions, however, which suggests that larger contributions may be found at the (comparatively cheap) expense of introducing additional loops.

For instance, at two loops the photon vacuum polarization acquires contributions by inserting a Lorentzviolating graviton self-energy within a charged-fermion loop (fig. 3). Assuming all charged fermions to be branebound, and so 4-dimensional, we estimate the following results for the photon: $c_{\gamma}^{2}-1 \sim\left(\frac{\alpha}{4 \pi}\right)\left(c_{f}^{2}-1\right)$, giving

$$
\begin{aligned}
& c_{\gamma}^{2}-1 \sim\left(\frac{\alpha}{4 \pi}\right)\left(\frac{\epsilon}{(4 \pi)^{[d / 2]}}\right)\left(\frac{m_{f}}{M_{d}}\right)^{d-2} \\
& \sim\left\{\begin{array}{llll}
1 \times 10^{-10} & \left(\epsilon \times 10^{3}\right) & \left(\mathrm{TeV} / M_{4}\right)^{2} & (d=4) \\
2 \times 10^{-11} & \left(\epsilon \times 10^{3}\right) & \left(\mathrm{TeV} / M_{5}\right)^{3} & (d=5) \\
3 \times 10^{-13} & \left(\epsilon \times 10^{3}\right) & \left(\mathrm{TeV} / M_{6}\right)^{4} & (d=6)
\end{array},\right.
\end{aligned}
$$

where $[d / 2]$ denotes the integer part of $d / 2$ and we use the heaviest known elementary particle, the top quark $\left(m_{t}=175 \mathrm{GeV}\right)$, for numerical purposes. Although this is still negligibly small for $d=4$, we shall see that the $d>$ 4 result can be large enough to provide new constraints on $\epsilon$ if $M_{d}$ is not too far above the $\mathrm{TeV}$ range.

A similar contribution arises to $b_{\gamma}$, although because of the weaker dependence on $m_{f}$ the price of a loop factor, $\alpha / 4 \pi$, is not worthwhile when $d=4$, where the direct one-loop result of eq. (32) is larger. We expect, then

$$
\begin{aligned}
b_{\gamma} M_{4}^{2} & =-\frac{304}{15}\left(\frac{\epsilon}{8 \pi}\right)^{2} \quad(d=4) \\
& =-3 \times 10^{-8}\left(\epsilon \times 10^{3}\right)^{2}
\end{aligned}
$$

in four dimensions, where the numerical result uses the conservative estimate $\log \left(\Lambda^{2} / \mu^{2}\right) \sim 1$.

For $d>4$ we estimate the two-loop result by $b_{\gamma} \sim$ $\left(\frac{\alpha}{4 \pi}\right) b_{f}$, and so find:

$$
\begin{aligned}
& b_{\gamma} M_{d}^{2} \sim\left(\frac{\alpha}{4 \pi}\right)\left(\frac{\epsilon^{2}}{(4 \pi)^{[d / 2]}}\right)\left(\frac{m_{f}}{M_{d}}\right)^{d-4} \quad(d>4)
\end{aligned}
$$

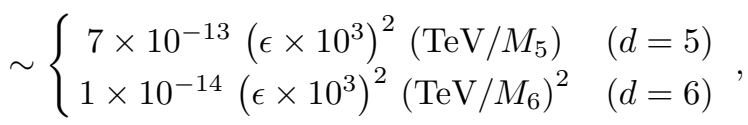

with $m_{t}$ being used for the fermion mass. The sign of $b_{\gamma}$ and $c_{\gamma}^{2}-1$ are not determined by these estimates (although they are certainly calculable within a more careful evaluation of Fig. (3).

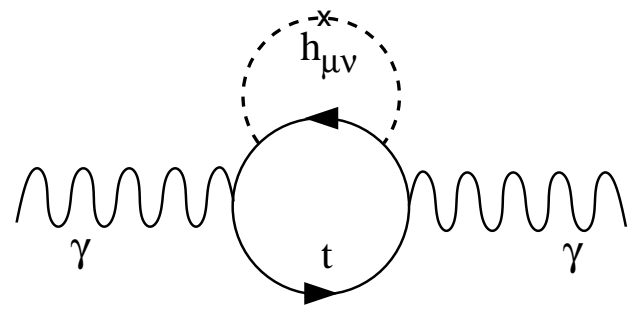

Figure 3. Two loop contribution to photon vacuum polarization from top quark and graviton.

\section{B. Electrons}

The next-cleanest application of the above analysis is to electrons, since so far as we know these are elementary and so our earlier results may be directly applied. Again taking the conservative estimate $\log \left(\Lambda^{2} / \mu^{2}\right) \sim 1$, we find that the direct graviton-loop contribution to the electron dispersion relation is

$$
\begin{aligned}
c_{e}^{2}-1 & \sim \frac{\epsilon}{(4 \pi)^{[d / 2]}}\left(\frac{m_{e}}{M_{d}}\right)^{d-2}, \\
b_{e} & \sim \frac{\epsilon^{2}}{(4 \pi)^{[d / 2]} M_{d}^{2}}\left(\frac{m_{e}}{M_{d}}\right)^{d-4},
\end{aligned}
$$

where $d$ counts the number of spacetime dimensions seen by the graviton within the effective theory at scales $\mu<$ $\Lambda \sim 1 \mathrm{TeV}$.

This should be compared with the result of inserting more massive particles into higher loops. For instance, a loop with a $\mathrm{W}$ boson and neutrino, with the graviton coupling to the $\mathrm{W}$ (fig. 4) gives the alternative contribution

$$
\begin{aligned}
& c_{e}^{2}-1 \sim\left(\frac{\alpha_{w}}{4 \pi}\right)\left(\frac{\epsilon}{(4 \pi)^{[d / 2]}}\right)\left(\frac{m_{w}}{M_{d}}\right)^{d-2}
\end{aligned}
$$

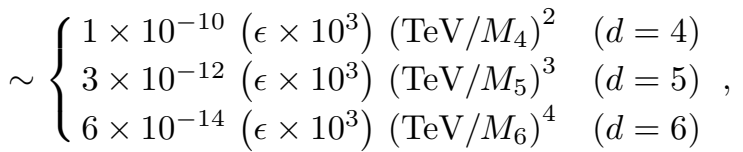

using $m_{w}=80 \mathrm{GeV}$, and $\log \left(\Lambda^{2} / m u^{2}\right)=1$. This last contribution (for $d \geq 4$ ) is always larger than the direct one, eq. (47), because $\left(m_{e} / m_{w}\right)^{d-2} \sim\left(6 \times 10^{-6}\right)^{d-2} \ll$ $\left(\alpha_{w} / 4 \pi\right) \sim 3 \times 10^{-3}$.

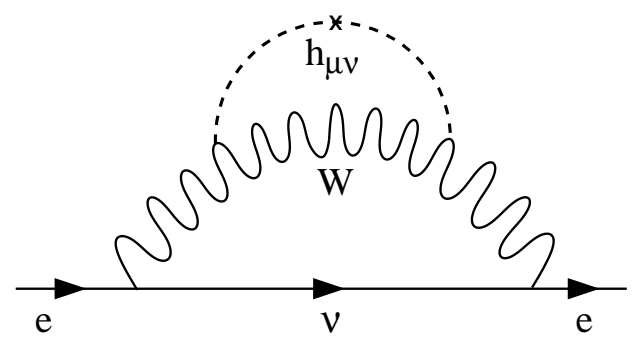

Figure 4. Two-loop contribution to electron self-energy from $\mathrm{W}$ boson and graviton, which can dominate over the one-loop graph with gravitons alone, Fig. 2. 
The higher-loop result can also provide a larger estimate for $b_{e}$, to wit:

$$
\begin{aligned}
& b_{e} M_{d}^{2} \sim\left(\frac{\alpha_{w}}{4 \pi}\right)\left(\frac{\epsilon^{2}}{(4 \pi)^{[d / 2]}}\right)\left(\frac{m_{w}}{M_{d}}\right)^{d-4} \quad(d \geq 5)
\end{aligned}
$$

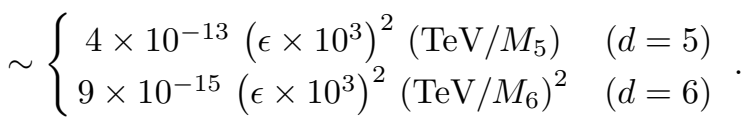

This dominates the direct one-loop result for all $d \geq 5$, because $\left(m_{e} / m_{w}\right)^{d-4} \sim\left(6 \times 10^{-6}\right)^{d-4} \ll\left(\alpha_{w} / 4 \pi\right) \sim$ $3 \times 10^{-3}$.

When $d=4$ the direct one-loop result wins, for which the results of the previous section give

$$
\begin{aligned}
b_{e} M_{4}^{2} & =-12\left(\frac{\epsilon}{8 \pi}\right)^{2} \quad(d=4) \\
& =-2 \times 10^{-8}\left(\epsilon \times 10^{3}\right)^{2} .
\end{aligned}
$$

\section{Protons}

The direct calculation of section III applies directly for the proton only within the effective theory below a $\mathrm{GeV}$, because it is only within this theory that the proton may be considered to be elementary. For higher scales the relevant degrees of freedom are quarks and gluons, for which we must estimate the size of Lorentz-violating effects.

The Lorentz-violating gluon and quark contributions may be estimated using our photon and electron results, eqs. (43) and (48), giving:

$$
\begin{aligned}
& c_{g}^{2}-1 \sim\left(\frac{\alpha_{s}}{4 \pi}\right)\left(\frac{\epsilon}{(4 \pi)^{[d / 2]}}\right)\left(\frac{m_{t}}{M_{d}}\right)^{d-2} \\
& c_{q}^{2}-1 \sim\left(\frac{\alpha_{w}}{4 \pi}\right)\left(\frac{\epsilon}{(4 \pi)^{[d / 2]}}\right)\left(\frac{m_{w}}{M_{d}}\right)^{d-2}
\end{aligned}
$$

where $\alpha_{s}$ is the QCD coupling, which we take to be 0.119 . From this we estimate the proton result to be

$$
\begin{aligned}
& c_{p}^{2}-1 \sim \operatorname{Max}\left(c_{g}^{2}-1, c_{q}^{2}-1\right) \\
& \sim\left(\frac{\alpha_{s}}{4 \pi}\right)\left(\frac{\epsilon}{(4 \pi)^{[d / 2]}}\right)\left(\frac{m_{t}}{M_{d}}\right)^{d-2} \\
& \sim\left\{\begin{array}{cccc}
2 \times 10^{-9} & \left(\epsilon \times 10^{3}\right) & \left(\mathrm{TeV} / M_{4}\right)^{2} & (d=4) \\
3 \times 10^{-10} & \left(\epsilon \times 10^{3}\right) & \left(\mathrm{TeV} / M_{5}\right)^{3} & (d=5) \\
4 \times 10^{-12} & \left(\epsilon \times 10^{3}\right) & \left(\mathrm{TeV} / M_{6}\right)^{4} & (d=6) .
\end{array}\right.
\end{aligned}
$$

The loop contributions to $b_{p}$ can also dominate for large numbers of dimensions, but not for $d=4$. We have the higher-loop contribution

$$
\begin{aligned}
& b_{p} M_{d}^{2} \sim\left(\frac{\alpha_{s}}{4 \pi}\right)\left(\frac{\epsilon^{2}}{(4 \pi)^{[d / 2]}}\right)\left(\frac{m_{f}}{M_{d}}\right)^{d-4} \quad(d \geq 5)
\end{aligned}
$$

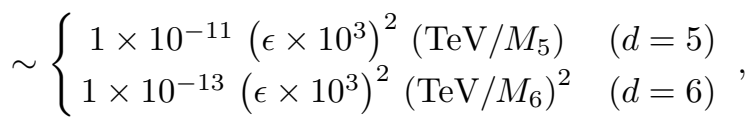

when $m_{f}=m_{t}$.

By contrast, for $d=4$ it is the direct one-loop result which dominates. In this case we have a result for fermions which is largely insensitive to the fermion mass:

$$
\begin{aligned}
b_{f} M_{4}^{2} & =-12\left(\frac{\epsilon}{8 \pi}\right)^{2} \quad(d=4) \\
& =-2 \times 10^{-8}\left(\epsilon \times 10^{3}\right)^{2} .
\end{aligned}
$$

For low-energy gravitons in the effective theory below the proton mass, the proton may be considered to be elementary and eq. (54) can be directly applied to the proton itself.

For higher-energy gravitons, eq. (54) would instead be applied to the light quarks, and the result for the proton would be obtained by taking the matrix element for the resulting effective quark operator, such as $\mathcal{O}_{\text {eff }}=\epsilon^{2} u^{\mu} u^{\nu} u^{\alpha} u^{\beta} \bar{q} \gamma_{\mu} \partial_{\nu} \partial_{\alpha} \partial_{\beta} q$, within the proton. On dimensional grounds this would produce the same result as eq. (54), but potentially with a different numerical coefficient. For concreteness, when comparing with the observables we use eq. (54) including the numerical factor obtained for an elementary proton.

Notice that the above estimates suggest that so long as higher-loop contributions dominate, a hierarchy is to be expected: $\left|c_{p}^{2}-1\right| \gg\left|c_{\gamma}^{2}-1\right| \sim\left|c_{e}^{2}-1\right|$. The same would not be expected to apply for $\left|b_{p}\right|,\left|b_{\gamma}\right|$ and $\left|b_{e}\right|$ when $d=4$, however.

\section{EXPERIMENTAL CONSTRAINTS}

We now turn to the experimental constraints which can be imposed on the quantities $c^{2}-1$ and $b$. Because we are interested in order-of-magnitude bounds, we derive constraints on each of these quantities as if they had arisen in the absence of the other. That is, we consider bounds on $c^{2}-1$ while taking $b=0$ and vice versa. This is justified unless there is an unnatural cancellation between the contributions of these quantities to physical observables.

\section{A. Existing Bounds on $c_{f}^{2}-c_{\gamma}^{2}$}

Good bounds exist on a difference between the propagation speeds of fermions and photons. Among those which do not depend on the sign of $c_{f}-c_{\gamma}$ are [9]: 


$$
\begin{aligned}
& \left|c_{f}-c_{\gamma}\right|<6 \times 10^{-22} \quad \text { Atomic spectroscopy } \\
& \left|c^{\prime}-c\right|_{\mu e}<4 \times 10^{-21} \quad \mu \rightarrow e \gamma \\
& \left|c_{K L}-c_{K S}\right|<3 \times 10^{-21} \quad K-\bar{K} \text { oscillations. }
\end{aligned}
$$

In the second bound $c^{\prime}$ denotes a particular combination of the Lorentz-violating couplings in flavor space, whose details are not important in what follows.

Although these bounds apply to both signs of $c^{2}-c_{\gamma}^{2}$, they are also subject to specific assumptions which need not apply to our calculation. For instance the $\mu \rightarrow e \gamma$ bound assumes the existence of Lorentz-violating terms which also violate lepton number, while the bounds involving neutral kaons require strangeness violation in addition to Lorentz-violation. Since the loops which would produce both type of flavor-symmetry violations from Lorentz-violation in the graviton sector are further suppressed by masses and mixing angles, we do not consider these bounds further.

The bounds from atomic spectroscopy are usually derived under the assumption that all matter particles share the same maximum propagation speed which differs from that of the photon - i.e., $c_{f} \neq c_{\gamma}$ is independent of $f$. Nevertheless these bounds are likely also to apply if $c_{f}$ differs for electrons and nucleons. Although we use the bound of ref. [9] in what follows, we regard a more careful analysis of constraints which are implied by these experiments to be worth pursuing.

If we apply these bounds directly to protons, we find $\left|c_{p}^{2}-c_{\gamma}^{2}\right|<6 \times 10^{-22}$. Using our previous estimates for the proton, eq. (53), and our expectation (see above) that $c_{p}^{2}$ differs from unity by more than does $c_{\gamma}^{2}$, we find comparison with the bound implies,

$$
\epsilon<\left(\frac{4 \pi}{\alpha_{s}}\right)(4 \pi)^{[d / 2]}\left(\frac{M_{d}}{m_{t}}\right)^{d-2} 10^{-21} .
$$
if

This is stronger than the direct bound $\epsilon<10^{-6}$ 网 only

$$
\frac{M_{d}}{\mathrm{TeV}}<0.2\left[\frac{10^{13}}{(4 \pi)^{[d / 2]}}\right]^{1 /(d-2)} \sim\left\{\begin{array}{cc}
3 \times 10^{4} & (d=4) \\
700 & (d=5) \\
46 & (d=6)
\end{array}\right.
$$

The bound so obtained is not useful for $d=4$. With extra dimensional gravitons the bound becomes useful only when $M_{d}$ is very small. It is only of borderline interest for the ADD scenario, for which $d=6$ but $M_{6}>50$ $\mathrm{TeV}$ is required from stellar-cooling bounds [24]. (We do not quote here the stronger limits coming from the nonobservance of gamma-ray decay products [25], or which

\footnotetext{
${ }^{\dagger \dagger}$ Using the weaker bound $\epsilon<10^{-3}$ makes the limits for $M_{d}$ larger by a factor of $10^{3 /(d-2)}$.
}

apply for supersymmetric models [26], because these may be evaded depending on model-dependent details.)

The bound is competitive, however, for the warped $5 \mathrm{D}$ model described below eq. (42), where $M_{5}$ is effectively the $\mathrm{TeV}$ scale.

\section{B. Čerenkov Bounds on $b_{f}$ and $c_{f}^{2}-1$}

We next consider constraints from high-energy cosmic rays, typically protons or photons. Their observation precludes the existence of processes which would tooefficiently deplete the energy of these particles. Since the particles involved are at higher energies, these processes are more sensitive to the higher-momentum coefficients, $b_{f}, b_{\gamma}$, than are low-energy laboratory limits.

Lorentz-violation can introduce dangerous processes by allowing decays in vacuum of the form $p \rightarrow p \gamma$ or $\gamma \rightarrow e^{+} e^{-}$or $\gamma \rightarrow p \bar{p}$. Such decays are precluded by energy and momentum conservation in Lorentz-invariant systems, but are allowed given Lorentz-violating dispersion relations. For instance, the process $p \rightarrow p \gamma$ becomes allowed if the dispersion relation raises the energy of the proton at a given momentum more than it does for the photon. Similarly, the process $\gamma \rightarrow e^{+} e^{-}$can occur if the photon energy is raised more than the electron's at a given momentum.

As applied to changes in the maximum propagation speed, the resulting bounds are therefore one-sided, in the sense that they are only relevant for one sign of $c_{p}-$ $c_{\gamma}$ or $c_{\gamma}-c_{e}$. For protons the strong one-sided bound obtained from $p \rightarrow p \gamma$ requires $c_{p}>c_{\gamma}$. Similarly, for $\gamma \rightarrow e^{+} e^{-}$or $\gamma \rightarrow p \bar{p}, c_{e}<c_{\gamma}$ and $c_{p}<c_{\gamma}$ are required to use the one-sided bound.

Keeping in mind that our estimates of the previous sections imply $\left|\delta c_{p}^{2}\right| \gg\left|\delta c_{\gamma}^{2}\right| \sim\left|\delta c_{e}^{2}\right|$, we find the limits obtained in this way for electrons and protons are

$$
\begin{aligned}
-2 \times 10^{-8}<c_{p}^{2}-c_{\gamma}^{2} \sim c_{p}^{2}-1 & <2 \times 10^{-23} \\
c_{e}^{2}-c_{\gamma}^{2} & >-6 \times 10^{-15} .
\end{aligned}
$$

In order of magnitude, these bounds are obtained by demanding that $\left|c_{f}^{2}-c_{\gamma}^{2}\right|<m_{f}^{2} / E^{2}$ where $m_{f}$ is the relevant fermion mass, $E$ is the energy of the observed cosmic ray, and the bound only applies to the appropriate sign of $c_{f}^{2}-c_{\gamma}^{2}$. The bound from $\gamma \rightarrow f \bar{f}$ is weaker than that from $p \rightarrow p \gamma$ because the most energetic cosmic ray proton has $E \sim 10^{8} \mathrm{TeV}$, while the most energetic observed gamma ray has $E \sim 50 \mathrm{TeV}$.

A similar bound also applies to the parameter $b_{f}$, which controls the dispersive part of the dispersion relation, eq. (21). For $p \rightarrow p \gamma$ the bound then applies only if $b_{p}>b_{\gamma}$ and for $\gamma \rightarrow f \bar{f}$ it requires $b_{f}<b_{\gamma}$. When the bound applies, it is of order $\left|b_{f}-b_{\gamma}\right|<m_{f}^{2} / E^{4}$.

We find in this way the constraints 


$$
\begin{aligned}
-\left(5 \times 10^{8} \mathrm{GeV}\right)^{-2}< & b_{p}-b_{\gamma}< \\
b_{e}-b_{\gamma} & >-\left(1 \times 10^{22} \mathrm{GeV}\right)^{-2} \\
& \left(10^{12} \mathrm{GeV}\right)^{-2} .
\end{aligned}
$$

$$
\text { 1. } b_{p}>b_{\gamma}
$$

Consider first the case $b_{p}>b_{\gamma}$, for which the best bound applies. For $d>4$ we compare this to the orderof-magnitude of the higher-loop results, with the resulting expectation $\left|b_{p}\right| \gg\left|b_{\gamma}\right|$. Using eq. (53) for $b_{p}$, as discussed in the previous section, and assuming the sign of the result is the one relevant for the bound's applicability, we obtain the limit

$$
\epsilon<\left[\frac{(4 \pi)^{[(d+2) / 2]}}{\alpha_{s}}\left(\frac{M_{d}}{m_{t}}\right)^{(d-4)}\right]^{1 / 2}\left(\frac{M_{d}}{3 \times 10^{22} \mathrm{GeV}}\right) .
$$

This is an improvement over $\epsilon<10^{-6}$ if

$$
\begin{aligned}
\frac{M_{d}}{\mathrm{TeV}}< & {\left[3 \times 10^{13}(0.175)^{(d-4) / 2} \sqrt{\frac{\alpha_{s}}{(4 \pi)^{[d / 2]+1}}}\right]^{2 /(d-2)} } \\
< & \left\{\begin{array}{cl}
2 \times 10^{7} & (d=5) \\
9 \times 10^{4} & (d=6) \\
6 \times 10^{4} & (d=7) \\
800 & (d=8) \\
300 & (d=9) \\
70 & (d=10)
\end{array}\right.
\end{aligned}
$$

(An improvement on the bound $\epsilon<10^{-3}$ is obtained for $M_{d}$ which can be a factor $10^{6 /(d-2)}$ larger.) We see the bound can be competitive for all $d$, provided $M_{d}$ is in the lower end of its allowed range. It is particularly strong for the $\operatorname{ADD}(d=6)$ and warped $\operatorname{RS}(d=5)$ cases, for which $M_{d}$ is in the TeV range.

To apply the bound to $d=4$ we instead use the oneloop result, for which the numerical factors and sign are known from our previous calculation (provided that the high-energy quark contribution does not dominate that for the low-energy proton, as discussed above). Although $b_{p}$ which is obtained is negative, the bound nonetheless applies because for $d=4$ we know that $b_{\gamma}$ is also negative, with $b_{f}>b_{\gamma}(c . f$. eqs. (44) and (54)). 用 We find

$$
b_{p}-b_{\gamma}=10^{-8} \frac{\left(\epsilon \times 10^{3}\right)^{2}}{M_{4}^{2}}<\left(3 \times 10^{22} \mathrm{GeV}\right)^{-2}
$$

\footnotetext{
$\ddagger \ddagger$ In fact, at the energies under consideration the graviton should resolve the proton as a number of partons, each carrying a small momentum fraction $x \ll 1$. Since the importance of the dispersive term grows with momentum as $p^{2}$, there will be a suppression $\sim x_{\mathrm{av}}^{2}$. Hence, compared to the photon, the effective $b$ for the proton may be close to zero.
}

which, using $M_{4}=2 \times 10^{18} \mathrm{GeV}$, implies $\epsilon<7 \times 10^{-4}$ a result which is competitive with the terrestrial bound, $\epsilon<10^{-3}$, when $\epsilon>0$, but is not as good as the bound $\epsilon \lesssim 10^{-6}$ obtained from the conservation of angular momentum of the sun (see the footnote on page 5 ).

$$
\text { 2. } b_{p}<b_{\gamma}
$$

If $b_{p}<b_{\gamma}$, then the best bound comes from the process $\gamma \rightarrow p \bar{p}$, which does not give a bound even as good as $\epsilon<10^{-3}$ for any $d$ given the constraint $M_{d}>1 \mathrm{TeV}$.

$$
\text { C. } b_{e}<b_{\gamma}
$$

The bound when $b_{e}<b_{\gamma}$ is not strong enough to be interesting for $d=4$, so we consider only higher dimensions. Since the order-of-magnitude result for the electron has $\left|b_{e}\right| \sim\left|b_{\gamma}\right|$ we have

$$
b_{\gamma} \sim \frac{\epsilon^{2}}{(4 \pi)^{[d / 2]} M_{d}^{2}}\left(\frac{m_{t}}{M_{d}}\right)^{d-4}\left(\frac{\alpha}{4 \pi}\right),
$$

and so, if $b_{\gamma}-b_{e}>0$ the bound becomes:

$$
\epsilon<\left[\frac{(4 \pi)^{[d / 2]+1}}{\alpha}\left(\frac{M_{d}}{m_{t}}\right)^{(d-4)}\right]^{1 / 2}\left(\frac{M_{d}}{10^{12} \mathrm{GeV}}\right) .
$$

For $M_{d}>1 \mathrm{TeV}$ this is never better than $\epsilon<10^{-6}$, but it represents progress relative to $\epsilon<10^{-3}$ if

$$
\begin{aligned}
\frac{M_{d}}{\mathrm{TeV}} & <\left[10^{6}(0.175)^{(d-4) / 2} \sqrt{\frac{\alpha}{(4 \pi)^{[d / 2]+1}}}\right]^{2 /(d-2)} \\
& = \begin{cases}90 & (d=5) \\
10 & (d=6)\end{cases}
\end{aligned}
$$

which is only of interest for $5 \mathrm{D}$ warped scenarios.

\section{Direct Bounds on $b_{\gamma}$}

The observation of cosmologically distant gamma rays from gamma-ray bursters can provide a bound on $\left|b_{\gamma}\right|$ which is similar in strength to the one just obtained, but applies equally well to both signs of the result. Ref. [27] argues that the absence of dispersion in these signals provides a sensitivity to changes in the photon dispersion which can be as small as

$$
\left|b_{\gamma}\right|<\left(9 \times 10^{11} \mathrm{GeV}\right)^{-2},
$$

which would correspond to

$$
\epsilon<\left[\frac{(4 \pi)^{[d / 2]+1}}{\alpha}\left(\frac{M_{d}}{m_{t}}\right)^{(d-4)}\right]^{1 / 2}\left(\frac{M_{d}}{10^{11} \mathrm{GeV}}\right) .
$$


This is better than $\epsilon<10^{-3}$ provided

$$
\begin{aligned}
& \frac{M_{d}}{\mathrm{TeV}}<\left[10^{5}(0.175)^{(d-4) / 2} \sqrt{\frac{\alpha}{(4 \pi)^{[d / 2]+1}}}\right]^{2 /(d-2)} \\
& =\left\{\begin{array}{cc}
20 & (d=5) \\
3 & (d=6)
\end{array}\right.
\end{aligned}
$$

which is comparable in strength to what was found above.

\section{CONCLUSIONS}

Brane-world scenarios allow the possibility of potentially strong preferred-frame effects arising from extradimensional bulk physics, and suggest these effects may be limited to the graviton sector. Depending on their sign, the magnitude of such changes to graviton dispersion can be comparatively large because of the graviton's extremely weak interactions. Motivated by this observation, we have explored how gravitational Lorentzviolation in a brane-world picture influences the properties of observable particles. We obtain the following results:

1. We analyze how Lorentz violation in extra dimensions arises within the low-energy four-dimensional field theory which is obtained once the extra dimensions are integrated out. We find the leading contributions to be the appearance of potentially different metrics in the kinetic terms which govern the propagation of the various low-energy particles. If preferred-frame effects dominate violations of translation or rotation invariance (in the preferred frame) then in flat space the metric seen by particle type ' $k$ ' may be written

$$
G_{\mu \nu}=\eta_{\mu \nu}+\left(1-c_{k}^{2}\right) u_{\mu} u_{\nu}
$$

where $u^{\mu}$ is the (approximately constant) 4-velocity which defines the preferred frame. $c_{k}$ may be interpreted as the maximum propagation speed of this particle type.

2. If subleading contributions at low energies are also included, then more complicated changes to the dispersion relation become possible. For low momenta these dispersion relations become $E_{k}^{2}=p^{2} c_{k}^{2}+b_{k} p^{4}+\ldots$ in the preferred frame. The next-to-leading coefficient $b_{k}$ causes dispersive propagation if it is nonzero. (Odd powers of $p$, like $p^{3}$, typically do not arise in the dispersion relation since they are usually forbidden by selection rules like rotation invariance in the preferred frame.)

3. Strong observational constraints exist which preclude large differences between the values $c_{k}$ and $b_{k}$ for photons and other particles. The strongest of these come from the absence of a dependence on the Earth's velocity through space in atomic spectra, and from the absence of Čerenkov-like decays of very-high-energy cosmic rays.
4. We compute how graviton loops can bring the news of Lorentz violation in the graviton sector to other particles for which stronger constraints exist. We do so quite generally at low energies for the purely massless $4 \mathrm{D}$ graviton (the lowest KK mode). We also compute these loops for the entire KK tower of gravitons, in the approximation that the dominant contribution comes from gravitons whose wavelengths are much shorter than are the typical curvature scales of the extra-dimensional metric.

5. We find that one-loop contributions for photons from graviton-induced Lorentz violation are small. Keeping the finite and log-divergent parts, we find gravitons do not induce any change at all in the photon maximum propagation speed, $c_{\gamma}$. The purely $4 \mathrm{D}$ graviton induces a dispersive term, $b_{\gamma} \sim\left(c_{g}-1\right)^{2} / M_{p}^{2}$. The contribution of the rest of the KK graviton tower to the photon energy tends to be suppressed by further powers of the photon momenta, and so is not important at low energies. Unfortunately this makes the strong constraints on photon properties based on gamma-ray bursts and dispersion in quasar signals 27 largely irrelevant to this kind of Lorentz violation.

6. Using the same approximations, we find fermions acquire changes to their low-energy dispersion relations with an amount which varies strongly with the fermion's mass. Among the three most abundant particles in everyday life - electrons, protons and photons - this predicts by far the biggest effects for protons. Contributions to $c_{k}-1$ are linear in $c_{g}-1$ (if $c_{g}$ is the graviton maximum speed) while those to $b_{k}$ are quadratic in $c_{g}-1$.

7. The strong mass dependence makes the results very sensitive to the high-energy spectrum of the theory, since heavy particles embedded in higher loops can produce larger contributions to low-energy Lorentz-violating effects than do the direct graviton loops. An estimate of this effect using the top quark or $W$-boson as the heavy particle suggests that protons receive larger contributions than do photons or electrons.

8. We find that current atomic constraints on $c_{k}-1$ for observable particles can provide limits on $c_{g}-1$ which are competitive with the direct bound $c_{g}-1 \lesssim 10^{-6}$ arising from post-Newtonian corrections in the solar system, but only if $d=5$ or 6 and if the higher-dimensional Planck mass, $M_{d}$, is as close as possible to the $\mathrm{TeV}$ scale. For instance, for a warped $d=5$ model with $M_{5} \sim 10 \mathrm{TeV}$, we find $\left|c_{g}-1\right|<3 \times 10^{-15}\left(M_{5} / \mathrm{TeV}\right)^{3}$. For $c_{g}>1$ this is an improvement over the direct bound $c_{g}-1<10^{-6}$ provided $M_{5}<700 \mathrm{TeV}$.

8. We find that stronger constraints on $c_{g}-1$ arise from limits on $b_{k}-b_{\gamma}$, depending on the dimension of the extra-dimensional spacetime, and on how low the $d$ dimensional Planck scale, $M_{d}$, is. Lower values of $M_{d}$ lead to better bounds, which can go up to values of order $M_{d} \sim 10^{7} \mathrm{TeV}$ (for $d=5$ ), provided that $b_{p}>b_{\gamma}$. The sign of this quantity is important, because the bounds which are most constraining are those which are based 
on the absence of too-efficient energy-loss mechanisms for the highest energy cosmic rays (which we take to be protons), and the decay channel $p \rightarrow p \gamma$ is only open if $b_{p}>b_{\gamma}$. Strikingly, purely 4-dimensional graviton loops can give contributions with the right sign, and which are large enough to produce bounds which are of order $\epsilon<7 \times 10^{-4}$. This makes them comparable with those from obtained from terrestrial experiments and the binary pulsar.

Perhaps our most surprising result is that, in some regimes, graviton loops are already being constrained by observational data. This is yet another striking way in which the brane-world picture can run against pre-braneworld intuition.

\section{Acknowledgements}

We would like to acknowledge fruitful conversations with Debajyoti Chauduri, Maxim Pospelov and Xerxes Tata. C.B. wishes to acknowledge discussions with Detlef Nolte and Witold Skiba at an early point in this research. J.M. acknowledges discussions with A.Pomarol. The research of C.B., J.C. and E.F. has been supported in part by N.S.E.R.C. of Canada and F.C.A.R. of Québec. J.M acknowledges support by CICYT Research Project AEN99-0766, MCyT and the Theory Group of McGill University.

\section{APPENDIX A}

In this appendix we record the expressions for the Feynman rule for the photon-graviton vertex, as obtained from the Einstein-Maxwell action, eq. (26). One finds:

$$
\begin{aligned}
V^{\alpha \beta: \mu \nu}(P, Q)=( & \left.P^{\mu} Q^{\nu}+P^{\nu} Q^{\mu}\right) \eta^{\alpha \beta} \\
& +\left(P^{\beta} Q^{\alpha}-P \cdot Q \eta^{\alpha \beta}\right) \eta^{\mu \nu} \\
& -\left(P^{\mu} Q^{\alpha}-P \cdot Q \eta^{\mu \alpha}\right) \eta^{\nu \beta} \\
& -\left(P^{\beta} Q^{\mu}-P \cdot Q \eta^{\mu \beta}\right) \eta^{\nu \alpha} \\
& -P^{\nu} Q^{\alpha} \eta^{\mu \beta}-P^{\beta} Q^{\nu} \eta^{\mu \alpha}
\end{aligned}
$$

which has the required symmetry properties:

$$
V^{\alpha \beta: \mu \nu}(P, Q)=V^{\alpha \beta: \nu \mu}(P, Q)=V^{\beta \alpha: \mu \nu}(Q, P) .
$$

\section{APPENDIX B}

We here record in more detail the results of the fermion self-energy calculation, using a graviton loop in $d$ dimensions.

\section{A. $d=5$}

For five spacetime dimensions evaluation of the graviton loop gives

$$
\begin{aligned}
& \mathcal{A}_{5}=-m^{4} \lambda_{5}\left(\frac{25}{9}+\frac{74}{9} \epsilon+\frac{85}{9} \epsilon^{2}+\ldots\right), \\
& \mathcal{B}_{5}=-m^{3} \lambda_{5}\left(\frac{35}{18} \epsilon+\frac{839}{180} \epsilon^{2}+\ldots\right), \\
& \mathcal{C}_{5}=-m^{2} \lambda_{5}\left(\frac{25}{6} \epsilon+\frac{517}{60} \epsilon^{2}+\ldots\right), \\
& \mathcal{D}_{5}=-m \lambda_{5}\left(\frac{101}{30} \epsilon^{2}+\ldots\right), \\
& \mathcal{E}_{5}=-\lambda_{5}\left(\frac{29}{30} \epsilon^{2}+\ldots\right) .
\end{aligned}
$$

where $\lambda_{5}=\left(\kappa_{5} / 8 \pi\right)^{2}$. The result is finite in dimensional regularization because one-loop results in odd dimensions always are with this regularization scheme. This result agrees with the finite part as computed by directly cutting off the momentum integrals and ignoring the divergent terms (none of which are logarithmically divergent).

These lead to the dispersion relation of eq. (21):

$$
\begin{aligned}
c_{f}^{2}-1 & =m_{f}^{3} \lambda_{5}\left[\frac{110}{9} \epsilon+\frac{239}{9} \epsilon^{2}+\ldots\right], \\
b_{f} & =m_{f} \lambda_{5}\left[\frac{26}{3} \epsilon^{2}+\ldots\right]
\end{aligned}
$$

Notice that both of these results are positive (provided $\epsilon>0)$.

\section{B. $d=6$}

For six spacetime dimensions the graviton loop gives

$$
\begin{aligned}
& \mathcal{A}_{6}=-m^{5} \lambda_{6}\left(\frac{9}{4}+\frac{25}{4} \epsilon+\frac{53}{8} \epsilon^{2}+\ldots\right), \\
& \mathcal{B}_{6}=-m^{4} \lambda_{6}\left(\frac{6}{5} \epsilon+\frac{83}{30} \epsilon^{2}+\ldots\right), \\
& \mathcal{C}_{6}=-m^{3} \lambda_{6}\left(\frac{18}{5} \epsilon+\frac{107}{15} \epsilon^{2}+\ldots\right), \\
& \mathcal{D}_{6}=-m^{2} \lambda_{6}\left(\frac{34}{15} \epsilon^{2}+\ldots\right) \\
& \mathcal{E}_{6}=-m \lambda_{6}\left(\frac{4}{3} \epsilon^{2}+\ldots\right) .
\end{aligned}
$$

where $\lambda_{6}=2 \kappa_{6}^{2} /(8 \pi)^{3} \mathcal{L}$. As before $\mathcal{L}=\log \left(\Lambda^{2} / \mu^{2}\right)=$ $2 /(6-n)$, when evaluated with an ultraviolet cutoff, $\Lambda$, and in dimensional regularization.

These lead to the $d=6$ results: 


$$
\begin{aligned}
c_{f}^{2}-1 & =m_{f}^{4} \lambda_{6}\left[\frac{48}{5} \epsilon+\frac{99}{5} \epsilon^{2}+\ldots\right], \\
b_{f} & =m_{f}^{2} \lambda_{6}\left[\frac{36}{5} \epsilon^{2}+\ldots\right]
\end{aligned}
$$

Again both results are positive for $\epsilon>0$.

\section{C. $d=7$}

Next, seven spacetime dimensions:

$$
\begin{aligned}
& \mathcal{A}_{7}=m^{6} \lambda_{7}\left(\frac{49}{25}+\frac{26}{5} \epsilon+\frac{129}{25} \epsilon^{2}+\ldots\right), \\
& \mathcal{B}_{7}=m^{5} \lambda_{7}\left(\frac{21}{25} \epsilon+\frac{131}{70} \epsilon^{2}+\ldots\right), \\
& \mathcal{C}_{7}=m^{4} \lambda_{7}\left(\frac{49}{15} \epsilon+\frac{263}{42} \epsilon^{2}+\ldots\right), \\
& \mathcal{D}_{7}=m^{3} \lambda_{7}\left(\frac{59}{35} \epsilon^{2}+\ldots\right) \\
& \mathcal{E}_{7}=m^{2} \lambda_{7}\left(\frac{53}{35} \epsilon^{2}+\ldots\right) .
\end{aligned}
$$

where $\lambda_{7}=\kappa_{7}^{2} /\left[6(4 \pi)^{3}\right]$, and is finite when evaluated in dimensional regularization.

These lead to the $d=7$ results:

$$
\begin{aligned}
c_{f}^{2}-1 & =-m_{f}^{5} \lambda_{7}\left[\frac{616}{75} \epsilon+\frac{244}{15} \epsilon^{2}+\ldots\right], \\
b_{f} & =-m_{f}^{3} \lambda_{7}\left[\frac{32}{5} \epsilon^{2}+\ldots\right]
\end{aligned}
$$

Here both results are negative for $\epsilon>0$.

\section{D. $d=8$}

For eight spacetime dimensions we have

$$
\begin{aligned}
& \mathcal{A}_{8}=m^{7} \lambda_{8}\left(\frac{16}{9}+\frac{41}{9} \epsilon+\frac{77}{18} \epsilon^{2}+\ldots\right), \\
& \mathcal{B}_{8}=m^{6} \lambda_{8}\left(\frac{40}{63} \epsilon+\frac{173}{126} \epsilon^{2}+\ldots\right), \\
& \mathcal{C}_{8}=m^{5} \lambda_{8}\left(\frac{64}{21} \epsilon+\frac{239}{42} \epsilon^{2}+\ldots\right), \\
& \mathcal{D}_{8}=m^{4} \lambda_{8}\left(\frac{4}{3} \epsilon^{2}+\ldots\right) \\
& \mathcal{E}_{8}=m^{3} \lambda_{8}\left(\frac{34}{21} \epsilon^{2}+\ldots\right) .
\end{aligned}
$$

where $\lambda_{8}=2 \kappa_{8}^{2} /(8 \pi)^{4} \mathcal{L}$. As before $\mathcal{L}=\log \left(\Lambda^{2} / \mu^{2}\right)=$ $2 /(8-n)$, when evaluated with an ultraviolet cutoff, $\Lambda$, and in dimensional regularization.

These lead to the $d=8$ results:

$$
\begin{aligned}
c_{f}^{2}-1 & =-m_{f}^{6} \lambda_{8}\left[\frac{464}{63} \epsilon+\frac{890}{63} \epsilon^{2}+\ldots\right], \\
b_{f} & =-m_{f}^{4} \lambda_{8}\left[\frac{124}{21} \epsilon^{2}+\ldots\right]
\end{aligned}
$$

Here both results are negative for $\epsilon>0$.

\section{E. $d=9$}

For $d=9$ we have

$$
\begin{aligned}
& \mathcal{A}_{9}=-m^{8} \lambda_{9}\left(\frac{81}{49}+\frac{202}{49} \epsilon+\frac{181}{49} \epsilon^{2}+\ldots\right), \\
& \mathcal{B}_{9}=-m^{7} \lambda_{9}\left(\frac{99}{196} \epsilon+\frac{3755}{3528} \epsilon^{2}+\ldots\right) \\
& \mathcal{C}_{9}=-m^{6} \lambda_{9}\left(\frac{81}{28} \epsilon+\frac{2665}{504} \epsilon^{2}+\ldots\right) \\
& \mathcal{D}_{9}=-m^{5} \lambda_{9}\left(\frac{277}{252} \epsilon^{2}+\ldots\right) \\
& \mathcal{E}_{9}=-m^{4} \lambda_{9}\left(\frac{425}{252} \epsilon^{2}+\ldots\right)
\end{aligned}
$$

where $\lambda_{9}=\kappa_{9}^{2} /\left[15(4 \pi)^{4}\right]$.

These lead to the results:

$$
\begin{aligned}
c_{f}^{2}-1 & =m_{f}^{7} \lambda_{9}\left[\frac{333}{49} \epsilon+\frac{1245}{98} \epsilon^{2}+\ldots\right], \\
b_{f} & =m_{f}^{5} \lambda_{9}\left[\frac{39}{7} \epsilon^{2}+\ldots\right]
\end{aligned}
$$

Here both results are positive for $\epsilon>0$.

\section{F. $\mathrm{d}=10$}

Finally, for ten spacetime dimensions:

$$
\begin{aligned}
& \mathcal{A}_{10}=-m^{9} \lambda_{10}\left(\frac{25}{16}+\frac{61}{16} \epsilon+\frac{105}{32} \epsilon^{2}+\ldots\right), \\
& \mathcal{B}_{10}=-m^{8} \lambda_{10}\left(\frac{5}{12} \epsilon+\frac{103}{120} \epsilon^{2}+\ldots\right), \\
& \mathcal{C}_{10}=-m^{7} \lambda_{10}\left(\frac{25}{9} \epsilon+\frac{449}{90} \epsilon^{2}+\ldots\right), \\
& \mathcal{D}_{10}=-m^{6} \lambda_{10}\left(\frac{14}{15} \epsilon^{2}+\ldots\right) \\
& \mathcal{E}_{10}=-m^{5} \lambda_{10}\left(\frac{26}{15} \epsilon^{2}+\ldots\right) .
\end{aligned}
$$

where $\lambda_{10}=4 \kappa_{10}^{2} /\left[3(8 \pi)^{5}\right] \mathcal{L}$, and $\mathcal{L}=\log \left(\Lambda^{2} / \mu^{2}\right)=$ $2 /(10-n)$, when evaluated with an ultraviolet cutoff, $\Lambda$, and in dimensional regularization.

These lead to the $d=10$ results: 


$$
\begin{aligned}
c_{f}^{2}-1 & =m_{f}^{8} \lambda_{10}\left[\frac{115}{18} \epsilon+\frac{421}{36} \epsilon^{2}+\ldots\right], \\
b_{f} & =m_{f}^{6} \lambda_{10}\left[\frac{16}{3} \epsilon^{2}+\ldots\right]
\end{aligned}
$$

$\epsilon>0$ ensures that both results in this case are positive.

[1] P. Horava and E. Witten, Nucl. Phys. B 475, 94 (1996) hep-th/9603142; P. Horava and E. Witten, Nucl. Phys. B 460, 506 (1996) hep-th/9510209.

[2] I. Antoniadis, Phys. Lett. B 246, 377 (1990); J. D. Lykken, Phys. Rev. D 54, 3693 (1996) hepth/9603133; I. Antoniadis, N. Arkani-Hamed, S. Dimopoulos and G. R. Dvali, Phys. Lett. B 436, 257 (1998) hep-ph/9804398; N. Arkani-Hamed, S. Dimopoulos and G. R. Dvali, Phys. Lett. B 429, 263 (1998) hepph/9803315; N. Arkani-Hamed, S. Dimopoulos and G. R. Dvali, Phys. Rev. D 59, 086004 (1999) hep$\mathrm{ph} / 9807344$.

[3] E. Witten, Nucl. Phys. B 471, 135 (1996) hepth/9602070; K. Benakli, Phys. Rev. D 60, 104002 (1999) [hep-ph/9809582]; C. P. Burgess, L. E. Ibañez and F. Quevedo, Phys. Lett. B 447, 257 (1999) hep$\mathrm{ph} / 9810535$.

[4] L. Randall and R. Sundrum, Phys. Rev. Lett. 83, 3370 (1999) hep-ph/9905221.

[5] L. Randall and R. Sundrum, Phys. Rev. Lett. 83, 4690 (1999) hep-th/9906064.

[6] C. Csaki, J. Erlich and C. Grojean, Nucl. Phys. B 604, 312 (2001) hep-th/0012143; C. Csaki, J. Erlich and C. Grojean, gr-qc/0105114; C. Grojean, F. Quevedo, G. Tasinato and I. Zavala C., JHEP 0108, 005 (2001) hep-th/0106120.

[7] A. C. Davis, C. Rhodes and I. Vernon, JHEP 0111, 015 (2001) hep-ph/0107250.

[8] S. K. Lamoreaux, J. P. Jacobs, B. R. Heckel, F. J. Raab and E. N. Fortson, Phys. Rev. Lett. 57, 3125 (1986); R. S. Van Dyck, P. B. Schwinberg and H. G. Dehmelt, Phys. Rev. Lett. 59 (1987) 26; S. M. Carroll, G. B. Field and R. Jackiw, Phys. Rev. D 41, 1231 (1990); G. Gabrielse, D. Phillips, W. Quint, H. Kalinowsky, G. Rouleau and W. Jhe, Phys. Rev. Lett. 74 (1995) 3544; J. F. Wardle, R. A. Perley and M. H. Cohen, Phys. Rev. Lett. 79, 1801 (1997) astro-ph/9705142; R. Bluhm, V. A. Kostelecky and N. Russell, Phys. Rev. Lett. 82, 2254 (1999) hep-ph/9810269; V. A. Kostelecky and C. D. Lane, Phys. Rev. D 60, 116010 (1999) hep-ph/9908504; V. A. Kostelecky, hep-ph/9904467; R. Cowsik, G. Rajalakshmi and B. V. Sreekantan, Prepared for 26th International Cosmic Ray Conference (ICRC 99), Salt Lake City, Utah, 17-25 Aug 1999; D. Bear, R. E. Stoner, R. L. Walsworth, V. A. Kostelecky and C. D. Lane, Phys. Rev. Lett. 85, 5038 (2000) physics/0007049; D. F. Phillips, M. A. Humphrey, E. M. Mattison, R. E. Stoner, R. F. Vessot and
R. L. Walsworth, Phys. Rev. D 63, 111101 (2001) physics/0008230; J.M. Carmona and J.L. Cortes, Phys. Lett. B494 (2000) 75-80, hep-th/0012028; T. Jacobson, S. Liberati and D. Mattingly, UMD preprint hep$\mathrm{ph} / 0112207$.

[9] S. R. Coleman and S. L. Glashow, Phys. Lett. B 405, 249 (1997) hep-ph/9703240; Phys. Rev. D 59, 116008 (1999) hep-ph/9812418.

[10] C. M. Will, Theory and Experiment in Gravitational Physics, 2nd Edition, (Cambridge University Press, 1993).

[11] For a recent summary of experimental bounds on deviations from General Relativity, see C. M. Will, grqc/9811036 C. M. Will, Living Rev. Rel. 4, 4 (2001) gr-qc/0103036.

[12] P. M. Mcculloch, J. H. Taylor and L. A. Fowler, In *Perth 1979, Proceedings, Gravitational Radiation, Collapsed Objects and Exact Solutions*, 5-11.(See Conference Index); J.H. Taylor and P.M. McCulloch, Ann. N.Y. Acad. Sci. (1980) 442; J.H. Taylor and J.M. Weisberg, Ap. J. (1982) 908.

[13] S. Carlip, Phys. Lett. A 267, 81 (2000) gr-qc/9909087.

[14] M. Nagano and A. A. Watson, Rev. Mod. Phys. 72, 689 (2000); F. Halzen, R. A. Vazquez, T. Stanev and H. P. Vankov, Astropart. Phys. 3, 151 (1995).

[15] G. D. Moore and A. E. Nelson, JHEP 0109, 023 (2001) hep-ph/0106220.

[16] R. R. Caldwell and D. Langlois, Phys. Lett. B 511, 129 (2001) gr-qc/0103070; E. Abdalla, B. Cuadros-Melgar, S. S. Feng and B. Wang, hep-th/0109024.

[17] J. W. Moffat, Int. J. Mod. Phys. D 2, 351 (1993) grqc/9211020; M. A. Clayton and J. W. Moffat, Phys. Lett. B 506, 177 (2001) gr-qc/0101126; D. J. Chung and K. Freese, Phys. Rev. D 62, 063513 (2000) hepph/9910235; Phys. Rev. D 61, 023511 (2000) (hepph/9906542; A. Albrecht and J. Magueijo, Phys. Rev. D 59, 043516 (1999) astro-ph/9811018; P. P. Avelino and C. J. Martins, Phys. Lett. B 459, 468 (1999) astroph/9906117]; S. Liberati, B. A. Bassett, C. Molina-Paris and M. Visser, Nucl. Phys. Proc. Suppl. 88, 259 (2000) astro-ph/0001481; J. D. Barrow and J. Magueijo, Class. Quant. Grav. 16, 1435 (1999) astro-ph/9901049.

[18] S. Weinberg, Gravitation and Cosmology, John Wiley \& Sons, 1972.

[19] K. Nordtvedt, Astrophys. J. 320 (1987) 871.

[20] C. P. Burgess and D. London, Phys. Rev. Lett. 69 (1992) 3428; Phys. Rev. D 48 (1993) 4337 hep-ph/9203216.

[21] G. F. Giudice, R. Rattazzi and J. D. Wells, Nucl. Phys. B 544, 3 (1999) hep-ph/9811291.

[22] T. Han, J. D. Lykken and R. J. Zhang, Phys. Rev. D 59, 105006 (1999) hep-ph/9811350.

[23] C. D. Hoyle, U. Schmidt, B. R. Heckel, E. G. Adelberger, J. H. Gundlach, D. J. Kapner and H. E. Swanson, Phys. Rev. Lett. 86, 1418 (2001) hep-ph/0011014.

[24] S. Cullen and M. Perelstein, Phys. Rev. Lett. 83, 268 (1999) arXiv:hep-ph/9903422; V. D. Barger, T. Han, C. Kao and R. J. Zhang, Phys. Lett. B 461, 34 (1999) arXiv:hep-ph/9905474; C. Hanhart, J. A. Pons, D. R. Phillips and S. Reddy, the size of large compact dimensions," Phys. Lett. B 509, 1 (2001) arXiv:astroph/0102063; S. Hannestad and G. Raffelt, Phys. Rev. 
Lett. 87, 051301 (2001) arXiv:hep-ph/0103201.

[25] L. J. Hall and D. R. Smith, Phys. Rev. D 60, 085008 (1999) arXiv:hep-ph/9904267; N. Arkani-Hamed, S. Dimopoulos and G. R. Dvali, sub-millimeter dimensions and TeV scale quantum gravity," Phys. Rev. D 59, 086004 (1999) arXiv:hep-ph/9807344.

[26] D. Atwood, C. P. Burgess, E. Filotas, F. Leblond, D. London and I. Maksymyk, Phys. Rev. D 63, 025007 (2001) arXiv:hep-ph/0007178.

[27] J. R. Ellis, K. Farakos, N. E. Mavromatos, V. A. Mitsou and D. V. Nanopoulos, Astrophys. J. 535, 139 (2000) astro-ph/9907340. 